\title{
HARALD WOHLRAPP
}

\section{Argumente stehen in einem Text nicht wie Gänseblumen in der Wiese herum}

\author{
Eine Kritik an Katharina Sobotas \\ empirischer Rechtstextanalyse
}

\section{Einleitung}

Es ist eine verbreitete Ansicht, dass Sprache und Denkweise der Juristen trocken, nüchtern und besonders rational seien. Das dürfte wohl mit der Erwartung zusammenhängen, dass die Bestimmung von Recht und Gerechtigkeit, gerade weil es hier um Sachverhalte geht, die von starken und stärksten Emotionen getragen sind, sich selbst aller gefühlsmäßigen Beteiligung enthalten sollte. Nun ist immerhin denkbar, dass es sich dabei um ein Postulat handelt, das der Jurisprudenz von außen, von den Anhängern einer missverstandenen Moderne, auferlegt worden ist, ein Postulat, von dem aber Insider wissen, dass es sich gar nicht erfüllen lässt, ja, mehr noch, dass eigentlich auch der Versuch seiner Erfüllung nicht wünschenswert wäre, weil dadurch das Recht zu einem Hort weltfremder Ideale würde. Ein Wahrnehmen der tatsächlichen Rechtspraxis mit ihrer lebensweltlichen Emotionalität und ihrer traditionseingebetteten Wandelbarkeit würde auf diese Weise durch die Illusion einer zeitlosen Verstandesreinheit verfälscht und vereitelt.

Soweit dieser Gedanke berechtigt ist, wäre es verdienstvoll, ja es wäre eine Aufgabe recht verstandener Aufklärung, den rationalen Mythos, der das Recht umgibt, aufzudecken und die Wirklichkeit, wie sie ist, darzustellen. (Ob diese dann so bleiben sollte, wäre natürlich anschlieBend durchaus noch zu fragen.)

Mit dieser möglicherweise aufklärerischen Aufgabe ist in etwa das Anliegen umschrieben, für das sich die Rechtstheoretikerin Katharina Sobota vor ca. anderthalb Jahrzehnten einzusetzen begann. Sie hat da$\mathrm{zu}$ einiges veröffentlicht; drei Studien, die ihre Arbeiten zum Thema darstellen, habe ich studiert, sie sind die Basis dieser kritischen Ausein- 
andersetzung. ${ }^{1}$ In den Arbeiten sind Methoden entwickelt worden, um juristische Texte, besonders höchstrichterliche Urteilsbegründungen, auf Rationalität, Emotionalität und Traditionsbindung zu untersuchen. Dabei kommt heraus, dass die Rationalität keineswegs überwiegt, sondern dass, im Gegenteil, gerade die wichtigsten, weil rechtsfortbildenden Passagen in solchen Urteilen sich weniger durch rationale Argumente als durch Gefühlsappell und Traditionseinbettung auszeichnen. Die Analysemethoden sind nun, und das ist das Besondere und Erstaunliche an Sobotas Arbeit, nicht die einer hermeneutisch-interpretatorischen Textarbeit, sondern sie sind empirisch. Es werden also Daten erhoben, es wird gezählt und gemessen, dann wird ausgewertet und erklärt, was man gefunden hat. Dieser Ansatz ist es, der mein skeptisches Interesse erregt. Gewiss können wir in Texten rationale von nichtrationalen Aspekten unterscheiden und unter Umständen auch ein Überwiegen der Letzteren konstatieren. Solange das umgangssprachlich-intuitiv, also ohne einen wissenschaftlichen Anspruch auf intersubjektive Nachprüfbarkeit geschieht, ist es harmlos. Es geht dann um subjektive Eindrücke und Anmutungen, die mehr oder weniger berechtigt sein können. Sobota behauptet aber, sie könne die rationalen Anteile eines Textes im Verhältnis zu den nichtrationalen regelrecht quantitativ bestimmen, habe sie gemessen und habe festgestellt, dass in den höchstrichterlichen Urteilsbegründungen gerade an den entscheidenden Stellen die Letzteren überwiegen.

Wenn das möglich ist, dann lässt sich eine Menge "weicher" text-, diskurs- und argumentationsanalytischer Arbeit, deren Wege mühsam und deren Resultate fallibel sind, einsparen bzw. durch harte, objektive Empirie ersetzen. Es fragt sich aber: Ist das wirklich möglich? Können wir Argumentation im Verhältnis zum Gefühlsappell quantitativ erfassen? Ist das, was in Sobotas Studien vorgeführt wird, empirische Forschung? Dieser Frage werde ich in der folgenden Schrift nachgehen. Ich werde dazu zunächst Sobotas Arbeiten und Ergebnisse referieren. An-

\footnotetext{
${ }^{1}$ KathaRina SOBOTA, Rhetorisches Seismogramm - eine neue Methode in der Rechtswissenschaft, in: Juristenzeitung 1992, 231-237; KATHARINA SOBOTA/OTTO BENKERT/ HANS MATHIAS KEPPLINGER, Psychopharmaka im Widerstreit. Eine Studie zur Akzeptanz von Psychopharmaka - Bevölkerungsumfrage und Medienanalyse. Berlin 1995; KATHARINA SOBOTA, Argumente und stilistische Überzeugungsmittel in Entscheidungen des Bundesverfassungsgerichts. Eine Rhetorik-Analyse auf empirischer Grundlage, in: Jahrbuch Rhetorik 15 (1996), 115-136. Die jüngste dieser Studien ist acht Jahre alt, aber die darin vorgestellten Auffassungen werden von der Autorin, inzwischen Katharina Gräfin von Schlieffen, weiterhin vertreten, so etwa auf dem Symposium „Juristische Argumentation" vom 8.-10. November 2002 in Schloss Blankensee; vgl. auch ihren Beitrag in diesem Band, den ich für meine Auseinandersetzung nicht mehr berücksichtigen konnte. Um möglichst klarzustellen, dass meine Kritik nicht auf die Person, sondern auf Texte zielt, habe ich deren Autorin hier durchgehend bei ihrem damaligen Namen benannt.
} 
schließend präsentiere ich eine kritische Auseinandersetzung mit dem Ansatz und zwar zunächst mit dem begrifflichen Fundament und dann mit den wissenschaftstheoretischen Vorstellungen. Als Hauptschwäche wird sich ein untauglicher und veralteter Argumentationsbegriff zeigen. Insgesamt demonstriert die Kritik, dass Sobotas Anspruch auf empirische Erfassung der rhetorischen und der argumentativen Aspekte von Texten zu Unrecht erhoben wird. Den Schluss des Aufsatzes bildet ein Ausblick, in dem der wahre Kern des Ansatzes herausgestellt und einige Hinweise $\mathrm{zu}$ seiner aussichtsreicheren Entwicklung gegeben werden.

\section{Darstellung}

\subsection{Die 92er-Studie}

Schon in dieser ersten Arbeit formuliert Sobota ihr Anliegen, das Rationalitätsideal der Jurisprudenz zu überprüfen und, da die Überprüfung enttäuschend ausfällt, dieses Ideal in den "Bereich der juristischen Selbstdarstellung" zu verweisen. Sie möchte stattdessen eine "ehrliche", "bescheidenere" Rechtsreflexion in Gang zu setzen, welche die gefühlsappellativen, traditionalen und ,irrationalen" Seiten der Rechtspraxis besser zu würdigen weiß $(92 / 237) .^{2}$

Wie gesagt, hat sie zu diesem Zweck juristische Texte im Hinblick darauf untersucht, wie viel Lebenswirklichkeit und Emotionalität neben der auch von ihr durchaus als wahrnehmbar konzedierten Rationalität sich dort ausmachen lässt. Für derartige Untersuchungen wird bekanntlich ein theoretischer Apparat gebraucht, der das erfassen kann, woraufhin der Gegenstand analysiert werden soll. Woher kann ein solcher kommen? Wie lassen sich Gefühlsappell und Traditionseinbettung in Texten dingfest machen? Worin soll sich die Rationalität vergegenständlichen? Für die Erfassung der rationalen Seite glaubt Sobota einen Argument-Begriff zu brauchen, der die Argumente als einzeln zählbare Einheiten modelliert. Dies wird aber jetzt noch als zu schwierig befunden und daher einstweilen zurückgestellt:

"Der Grund dafür ist, dass in juristischen Texten die wenigsten Schritte einer argumentativen Gedankenfolge explizit erwähnt werden: das meiste wird nur angedeutet." (92/233)

\footnotetext{
${ }^{2}$ Die drei in Fußnote 1 genannten Studien werden hier im laufenden Text ständig zitiert und zwar in der Form: (Jahreszahl/Seitenzahl). (92/237) bedeutet also: KATHARINA SOBOTA, Rhetorisches Seismogramm etc., Seite 237.
} 
Für die emotionale Seite glaubt sie sich jedoch auf festem Boden zu befinden, denn hier kann sie sich auf die Rhetoriktradition stützen. Sie versteht die Kanones der traditionellen Rhetorik als Sammlungen von emotionsaffizierenden Text-Vorkommnissen, und weil sie in juristischen Texten offenbar eine erstaunliche Menge derartiger Vorkommnisse antrifft, konstruiert sie ihren Apparat zur Erfassung der emotionalen Textaspekte aus jenem rhetorischen Erbe. Sie systematisiert die überkommenen Sammlungen rhetorischer Topoi, Loci und Redeverzierungen für ihre speziellen Zwecke, indem sie 30 "rhetorische Figuren" auswählt, charakterisiert und soweit möglich als isolierbare TextVorkommnisse intersubjektiv identifizierbar $\mathrm{zu}$ machen sucht. Zur begrifflichen Bestimmung wird die rhetorische Figur abgegrenzt gegen das Argument. Während jenes „auf den ,Kopf' des Zuhörers zielt, wendet sich die Figur an sein "Herz"' (92/232). Um zu zeigen, was sie meint, bringt sie allerlei Beispiele von Wendungen aus Rechtstexten, in denen rhetorische Figuren vorkommen (ebd.). Das Charakteristikum des Herz- bzw. Gefühlsappells wird einigermaßen nachvollziehbar, wenn es sich um plastische Metaphern, Steigerungen, Übertreibungen, Wortumstellungen oder Wiederholungen (von Buchstaben oder Wörtern) handelt. Derartige Figuren würden, so formuliert sie es dann als regelrechte "Hypothese" (ebd.), "nicht nur ausnahmsweise (auftreten, H. W.), sondern ganz allgemein ein wichtiges juristisches Überzeugungsmittel darstellen" (ebd.). Die Verifikation dieser Hypothese soll darin bestehen, jene allgemeine Wichtigkeit als eine überdurchschnittliche Häufigkeit nichtrationaler Überzeugungsmittel in juristischen Texten erfahrbar zu machen. Um jedoch die Erwartungen des Auditoriums an eine empirische Verifikation ihrer Hypothese in die richtigen Bahnen zu lenken, räumt sie ein, dass die Figuren eher „ungewisse Muster" seien, die sich nur "annäherungsweise festlegen lassen" (ebd.). Dennoch sei es möglich, an Texten regelrechte Auszählungen durchzuführen und die Auszählergebnisse durch Bezugnahme auf Texteinheiten (500 Wörter, 1.000 Wörter, $1 / 2$ Seite) zu Maßzahlen für die „Figurendichte" bzw. die "rhetorische Intensität" (ebd.) einer Textpassage zu machen.

Mit diesem Instrument ausgerüstet, kann sie dann nicht nur in Rechtstexten, sondern in beliebigem Material, z. B. in Zeitungsartikeln, sogar der Regenbogenpresse, die rhetorische Intensität messen und Vergleiche anstellen. Es finden sich in der Tat merkwürdige und interessante Verhältnisse. Ich nenne die drei hervorstechendsten. Zum ersten: Die Texte des BVerfG, also eines doch wirklich ausgestellten Produzenten juristischer Prosa, weisen eine deutlich höhere rhetorische Intensität auf als die Artikel in Lokalzeitungen (92/233, Abb. 1). Dies 
muss jeden, der beim BVerfG mit besonderer Trockenheit und Nüchternheit gerechnet hatte, überraschen. Das zweite bedenkliche Ergebnis ist Folgendes: Die rhetorische Intensität der BVerfG-Texte ist in den verschiedenen Abschnitten (Leitsätze, Urteilskopf, Entscheidungsformel) verschieden hoch, die Höhenunterschiede lassen sich in Kurven darstellen. Wenn nun die für verschiedene Urteile, um ganz verschiedene Sachverhalte, ermittelten Kurven verglichen werden, dann zeigt sich, dass diese Kurven "alle ein gemeinsames Muster" haben (92/234). Dieses Muster wird von Sobota als die "rhetorische Handschrift des BVerfG" bezeichnet (ebd.). Die dritte Überraschung ist die beunruhigendste. Sie betrifft die Inhalte. Die Autorin stellt nämlich fest, "dass an denjenigen Stellen der Gründe, die nach allgemeiner juristischer Auffassung als die ,inhaltlich entscheidenden' angesehen werden dürften, der Figurenaufwand auffällig gesteigert wird" (92/235). Also nicht etwa größere logische Durchdringung, so erweisen diese "Messungen", nicht eine Zunahme an Deutlichkeit, Stärke, Anzahl der Argumente ist an den kritischen Stellen zu beobachten, sondern eine Zunahme der rhetorischen Intensität.

Von den gefundenen Forschungsergebnissen lässt sich Sobota zu weit reichenden Feststellungen verleiten: $\mathrm{Da}$ "das Streben nach Sinn, Gewissheit und Gerechtigkeit in eine Dimension verweist, die mit rationalen Mitteln nur ungenügend zu erfassen" sei (92/237), "(könnten) die ,irrationalen' Formen der Wirklichkeitskonstruktion, zu denen auch die rhetorischen Figuren zählen, ... hier tragfähiger sein als rationale Konstrukte" (ebd.). Würde das bedacht, dann sei ein Hintergrund eröffnet, vor dem der Stil des BVerfG ,nicht nur als adäquat, sondern auch als vorbildlich" erscheine (ebd.). Gleichwohl soll diese 1992 publizierte Studie aber "lediglich explorative, keine konfirmative Funktion" haben $(92 / 233)$.

\subsection{Die 95er-Studie}

Um aus dem Explorationsstadium herauszukommen, bearbeitet sie weiter ihr „Messgerät” für rhetorische Dichte und publiziert $1995 \mathrm{zu}-$ sammen mit zwei Koautoren eine Studie, in der Texte aus der Presseberichterstattung über Psychopharmaka untersucht werden. Hier werden jetzt erst einmal ausführliche Reflexionen zum Wesen und zur Funktion der "rhetorischen Figur" angestellt. Die Autoren schreiben:

„... gegen rhetorische Kunstgriffe scheint man (anders als bei Argumenten, H. W.) kaum etwas ausrichten zu können ... Vermutlich liegt das daran, dass rhetorische Figuren überwiegend an das Gefühl appellieren ... Wenn es auch nicht sinnvoll ist, zwischen Verstand und Gefühl eine genaue Trennungslinie zu ziehen, so kann man doch tendenziell sagen, dass sich gefühlsmäßige 
Dinge im Gegensatz zu Verstandesangelegenheiten nur schwer beobachten und noch schwerer mit Bewusstsein analysieren, kontrollieren und parieren lassen. Aus diesem Grund kann ein Autor über den Stil seiner Darstellung oft versteckte und damit besonders nachdrückliche Effekte erzielen. Der Leser ist dieser Beeinflussung ausgeliefert. Selbst wenn er die Art der Rhetorik begreift, hat sie schon gewirkt; man kann sie durch keine nachträgliche Analyse ungeschehen machen." (95/79)

Mit derartigen Beobachtungen ist, so ließe sich denken, doch wohl eines unmissverständlich klar gestellt: rhetorische Figuren sind nicht etwa Mittel der Klärung und Verdeutlichung in Texten, sondern der Beeinflussung und Manipulation. Wäre es also nicht eigentlich angebracht, den Gebrauch rhetorischer Figuren sehr kritisch zu sehen? Die Autoren finden das nicht:

"Unhaltbar wäre ... stilistisches Pathos ... als Versuch sachfremder Manipulation zu verurteilen. Stilmittel, selbst wenn sie allein der Affektsteigerung dienen, können einer Sache durchaus nützlich sein" (95/94)

Es werden vier nützliche Effekte angeführt: Die Aufmerksamkeit des Adressaten werde erhöht, die Botschaft werde besser behalten, die intendierten Wertungen würden emotional verstärkt und schließlich brauche man sich weniger um Argumente zu kümmern: „Wo der Autor Stilmittel häuft, muss er weniger argumentieren" (ebd.). Es klingt hier der besondere Argumentbegriff an, mit dem Sobota arbeitet. Argumente sind offenbar durch rhetorische Stilmittel ersetzbar. Beide scheinen also im Grunde dieselbe Funktion für einen Text zu haben. Und die rhetorischen Figuren scheinen diese Funktion einfacher, sicherer und aufwandsärmer wahrnehmen zu können als die Argumente. Um was für eine Funktion es sich dabei handelt, das lehrt der Oberbegriff "Überzeugungsmittel”. Doch was ist dabei „Überzeugen“? Ich werde dieser Frage unten im Abschnitt 3.1.3. nachgehen. An dieser Stelle bietet es sich an, die Aussage, der Adressat könne gegen die rhetorische Vereinnahmung "kaum etwas ausrichten", mit der anderen, dass es sich aber dennoch nicht um "sachfremde Manipulation" handele, zu kombinieren. Das liefe auf die Bestimmung hinaus, dass es beim rhetorischen „Überzeugen" um eine Art sachdienlicher Manipulation ginge. $\mathrm{Ob}$ dergleichen zu befürworten ist, das hängt sicherlich von der Sache $a b$, um die es dabei geht. Doch selbst wenn das eine "gute Sache" wäre, ist eine Methode, die den Betroffenen unbemerkt zu etwas veranlasst, nicht einfach gutzuheißen. Der Zweck heiligt nun einmal nicht die Mittel.

Was den theoretischen Apparat für die empirische Forschung betrifft, so ist der eigene Figurenkanon nun von 30 auf 37 Figuren erweitert. Es wird eine detaillierte Beschreibung mit exemplarischen Illustrationen publiziert (95/202-208) und anhand beigelegter Analysen eini- 
ger Zeitungsartikel gezeigt, wie die Figuren im Textmaterial identifiziert werden (95/82-83). Ferner werden innerhalb des "Pathos" - dies ist jetzt der Titel für die rhetorischen Figuren bzw. für deren unterstellte Wirkung - zwei verschiedene Qualitäten unterschieden. Es gibt jetzt "engagiertes, praktisches Pathos" (z. B. als Kommentar zu einer Krawatte: "Wie scheußlich!") und "raffiniertes, distanzierendes Pathos" (in der gleichen Situation: „Im Prinzip ist diese Krawatte nicht hässlich, sie passt nur nicht zu den roten Streifen in deinem Anzug.") (95/89)

Als generelles Resultat der Untersuchung wird gefunden: In Presseberichten über Psychopharmaka „häufen sich rhetorische Stilmittel, die den Leser gefühlsmäßig ansprechen. Bei einem anderen, vergleichbaren Thema (Mittel gegen Herz-Kreislauf-Erkrankungen) ist dies in deutlich geringerem Umfang der Fall" (95/93). Die durchschnittliche Figurendichte ist bei den Psychopharmaka 92, bei den Herz-Kreislauf-Mitteln ist sie $72(95 / 84)$. Zum Vergleich: Bei höchstrichterlichen Urteilen hatte Sobota eine durchschnittliche Figurendichte von 46,5 gefunden $(92 / 234)$. Was mit diesem Resultat also jedenfalls erreicht ist, das ist eine Konsolidierung ihrer Methode und eine gewisse Plausibilisierung ihrer Ergebnisse.

\subsection{Die 96er-Studie}

1996 wird eine weitere Publikation zur Thematik vorgelegt. Mittlerweile hat die rationale Rechtstheorie Verstärkung von der Philosophie bekommen, und zwar von der Diskurstheorie, also jener von Habermas und seinen Mitstreitern promovierten Lehre, die menschliche Vernunft artikuliere sich wesentlich im argumentierenden Diskurs; dieser sei der Ort der Entscheidung über die Vernünftigkeit jedweder sozial relevanter Problemlösung, und insbesondere auch der Auseinandersetzungen um den Rechtsstaat. ${ }^{3}$ Die Frage, wie viel von diesem Ansatz in welcher Weise für die Jurisprudenz fruchtbar zu machen sei, hat seither zahlreiche Diskussionen und Publikationen hervorgebracht. Für Sobota ist damit eine neue Stellung an der Front, an der sie streitet, eröffnet worden, und sie wirft sich mit Verve ins Gefecht. Da werden, schreibt sie, Systeme konstruiert, die

„.... existieren ... nur in der Theorie. Sie regulieren nichts außerhalb des Akademiebetriebs, sind also nicht, sozial effektiv'. Bewusst wenden sie sich von den gelebten Ordnungen $a b$ und entwerfen ein unempirisches Normenwerk, nach dessen Bild sich die Wirklichkeit formen soll." (96/117)

\footnotetext{
${ }^{3}$ Vgl. JÜRGEN HABERMAS, Faktizität und Geltung. Beiträge zur Diskurstheorie des Rechts und des demokratischen Rechtsstaats. Frankfurt am Main 1992.
} 
Das Ganze sei kantianischer Idealismus. Um mit der Theoriebildung wirklichkeitsnah zu bleiben, sei aber auf Aristoteles zurückzugreifen. Bei dem ginge es nämlich nicht um abstrakte Vernünftigkeit, sondern um Lebensklugheit, Bestimmung des konkret Angemessenen.

„Die Einübung ins Angemessene verlangt nicht nur ein Verstandestraining, sondern eine Schule der Gefühle ... Nicht nur Kognition entscheidet, sondern auch Moral und Tradition, Ästhetik und Emotion. Dieses Zusammenspiel erfasst Aristoteles mit der Trias ,Logos - Ethos - Pathos'." (96/118)

Die Entdeckung dieses "Zusammenspiels”, bzw. die Entwicklung der Kategorien "Logos" und „Ethos", das ist das Neue gegenüber den bisherigen Arbeiten und es erlaubt, die bisher produzierten Resultate ganz erheblich deutlicher $\mathrm{zu}$ formulieren und noch weit reichender $\mathrm{zu}$ interpretieren.

,Logos' ist der Titel für Text-Vorkommnisse, bei denen es um Argumente geht. Dabei wird "der Begriff Argument ... sehr weit verstanden" (96/120). Gleichwohl müssen Argumente eindeutig identifizierbar sein, denn sie sollen ja gezählt (und den rhetorischen Figuren gegenübergestellt) werden. Sobota erläutert ihre Definition wie folgt:

"Als Logos-Argument gelten Aussagen oder Andeutungen, die man ... nach dem Schema formulieren könnte, das ist so, weil ...'. Außerdem muss bei dieser Konstruktion - $x$, weil $X$ - das begründende $X$ zum behaupteten $x$ als die weitere Begrifflichkeit erscheinen, etwa wie sich eine Regel ... zu einer Einzelgegebenheit verhält." (96/120)

Es werden "Kodifikationsargumente", "Rechtsargumente" und "Alltagsargumente" unterschieden, dann noch „Folgerungen”, die zwar eigentlich "keine Argumente” seien, aber dennoch als solche gezählt würden "um sicherzugehen, dass alle Argumente erfasst wurden. Doppelbewertungen werden dabei in Kauf genommen" (ebd.). Was das Erfassen selbst betrifft:

„Jedes Argument zählt gleich. Für jede Begründung gibt es einen Punkt. Sind zwischen Behauptung und Begründung offenbar noch weitere Prämissen eingeschaltet, zählen diese nur, wenn sie im Text Anklang gefunden haben." $(96 / 120)$

Man ahnt, dass das Identifizieren so verstandener "Argumente” schwieriger und weniger eindeutig sein könnte, als man sich das vielleicht wünscht. Und in der Tat, dieses argumentative Geschehen kann

„nur von einem sachkundigen, gutwilligen Leser erfasst werden. Der Codierer muss mit juristischem Wissen, Intuition und Phantasie rekonstruieren, was die Richter vielleicht gemeint haben könnten." (ebd., Hervorhebung im Text)

Die zweite Kategorie ist „Ethos”. Der Ausdruck ist der Titel für Vorkommnisse, bei denen „auf die Sitten, die Institutionen und prägenden 
Wertvorstellungen" Bezug genommen wird (96/121). Ausdrücklich gemacht werden drei Arten solcher Bezüge: "Autoritätsargumente (dieses ist so, weil jener - eine respektable Person oder Institution - das sagt), Textargumente (dies ist so, weil es geschrieben steht) und Quellenangaben (dort genau steht es)" (ebd.). Nun können ja derartige Bezüge, selbst nach einem landläufigen Verständnis, durchaus "argumentativ" sein. Offenbar ist also "Ethos" eigentlich nur eine Untergruppe von "Logos", wobei der Traditionsbezug das Spezifikum sein soll. Wenn Sobota in ihren Schaubildern die Anteile von Logos und Ethos in Säulen übereineinder stapelt (die dann von der imposanten Zackenkette des Pathos-Gebirges überwölbt werden), dann wird auch in der Anschauung nahe gelegt, dass Logos und Ethos zusammen das argumentative Geschehen in einem Text erfassen sollen („Der y-Wert der Säulen entspricht der jeweiligen Häufigkeit von Logos und Ethos (=Argumentanteil)" (96/123). Ganz konsequent ist das aber eigentlich nicht, denn in der "Trias Logos - Ethos - Pathos" sollte ja "Ethos" eine Traditionseinbettung betiteln, die sich offenbar im Logos nicht darstellen könnte. Wozu brauchte es sonst überhaupt einen dritten Oberbegriff, der anscheinend gleichrangig neben "Logos" und "Pathos" steht? Sobota scheint dieses Problem zu sehen. Sie schreibt einräumend:

"Der hier verwendete Ethos-Begriff ist ein vorläufiges Konstrukt, um Messungen durchführen zu können. Folgt man der Rhetoriklehre, kann man annehmen, dass dadurch einige, aber keinesfalls alle Aspekte des juristischen Ethos beleuchtet werden." (96/121)

Die Frage, wie sich weitere Aspekte erfassen lassen, und zwar in zählbaren Einheiten, bleibt offen. Der Leser muss sich mit dem Eingeständnis der Vorläufigkeit dieser "Kategorie" begnügen und hoffen, dass sie keinen theoretischen Schaden anrichtet.

"Pathos" ist in dieser Arbeit der Titel für die rhetorischen Figuren, die jetzt charakterisiert werden als "nicht-argumentative Stilmittel" bzw. "Überzeugungsmittel, die nach den modernen, rationalistischen Rechtstheorien tabu sind" (96/121). Wie schon zuvor unterscheidet sie 37 verschiedene Figuren (die teilweise auch noch Varianten haben können). In der Darstellung dieser 96er-Studie zählt sie nochmals einige besonders eindrucksvolle auf, so dass die Frage nach ihrer möglichen Funktion wieder sehr nahe liegt. Von Alliteration, Inversion, Metapher ist es leicht nachvollziehbar, dass diese mit Affekten, Gefühlen, meinetwegen auch mit dem "Herzen" zu tun haben. Weit weniger einleuchtend ist das bei den an dieser Stelle (96/121 f.) auch angesprochenen Figuren der Differenzierung (einerseits - andererseits), der Restrictio (zwar - aber) oder der Polarisierung (Antithese). In welchem Sinne sollten die eher das Fühlen als das Verstehen ansprechen? Und 
selbst wenn das vorkommen mag, ist es nicht eher abhängig von den Inhalten, um die es dabei geht? Auch wenn diese Frage beantwortbar wäre, läge dahinter noch die Frage, in welchem Sinne derartige "Stilmittel" denn eigentlich "Überzeugungsmittel” sein sollen. Es ist nun einmal nicht alles, was gesagt und vorgetragen wird, überzeugend. Und selbst wenn ein Überzeugungszweck unterstellt werden könnte, ist noch lange nicht sicher, dass alles, was unter diesem $Z$ weck veranstaltet wird, ihm auch in der Tat dient. Auf diese Frage werde ich unten in der Kritik ausführlich zu sprechen kommen. Sobota bescheidet ihre Leserschaft einstweilen mit dem Hinweis, "das Verfahren der PathosFeststellung [habe sich] ... in einer Untersuchung an über 300 Texten als reliabel erwiesen". Zwischen der gemessenen Pathos-Intensität (d. h. also der Figurendichte) und der verspürten Emotionalität bestehe eine hohe Korrelation (0.93, an zwei Probanden gefunden, 96/122).

Die in der Untersuchung erzielten Ergebnisse bestätigen, differenzieren und vervollständigen die der vorangegangenen Studien. Das erste Resultat, dass also Pathos ein ganz übliches Überzeugungsmittel auch und gerade in der Jurisprudenz sei, das ist nun schon beinahe selbstverständlich. Das dritte Resultat, dass an den entscheidenden Stellen eine besondere Häufung von rhetorischen Figuren zu finden sei, wird aber jetzt, wegen der exakteren Konfrontationsmöglichkeit mit den argumentativen Vorkommnissen, schärfer formulierbar:

„In den eigentlichen Entscheidungsgründen, den eigenen Rechtserwägungen des Gerichts, tritt ... Logos in den Hintergrund. Die entscheidenden Stellen, die sich bei den gewichtigen Entscheidungen regelmäßig in den Leitsätzen wiederfinden, werden mit hohem und höchstem Pathos vorgetragen." $(96 / 122 \mathrm{f}$.

Ergänzend kann die Autorin im Hinblick auf das Verhältnis von Logos, Ethos und Pathos feststellen,

"dass Logos und Ethos zu Pathos in einem reziproken Verhältnis stehen: Viel Pathos - wenig Logos, wenig Pathos - viel Logos. Für den Einsatz von Ethos gilt dies mit der Maßgabe, dass nicht nur Pathos, sondern auch Logos den Verweis auf Autoritäten und Quellen entbehrlich macht." (96/123)

Was schließlich das zweite Resultat, also die Charakteristika der PathosKurven, betrifft, bekommt die in der 92er-Studie sogenannte "Handschrift des BVerfG" eine noch ausgeprägtere Gestalt. In der "typischen Überzeugungsstruktur eines Urteils" (96/130) zeigt sich die eben schon erwähnte Reziprozität von Logos bzw. Ethos und Pathos "so konsequent, dass man geradezu an eine Spiegelung (Krebsbewegung, Interferenz) denken muss" (96/125). Gegen Ende der Pathos-Kurve gibt es "den häufig zu beobachtenden Doppelgipfel (Twin Peak) ..., der wie üblich mit den entscheidenden Schritten der Gedankenführung zusam- 
menfällt" (96/124). Diese Gestalt der Pathos-Kurve scheint so augenfällig zu sein, dass einer von Sobotas Mitstreitern es für angemessen befunden hat, sie nach ihrer Entdeckerin als die "Sobota-Spule" zu bezeichnen. ${ }^{4}$

Mit diesen Resultaten ist sie sich der Vermutungen, die 1992 noch "explorativ" vorgestellt worden waren, so sicher, dass sie sie als Munition gegen die rationalistischen Rechtstheorien und besonders gegen Habermas sowie die „Postulate der Egalität, Modernität und Intellektualität” (96/133) richten kann: „Rechtssysteme sind weithin ,unmodern', nämlich rituell, symbolisch, traditional und hierarchisch strukturiert." (96/133) Doch irgendwie scheinen ihr dann doch Bedenken zu kommen, welchen Kräften sie da das Wort reden könnte. Jedenfalls wendet sie sich auch ausdrücklich gegen ein "Recht der Affekte" (96/135), stellt fest: „Rechtstexte sind deutlich logos-orientiert” (96/134) und warnt: „Ein Gerichtswesen, das sich nicht vorrangig auf kodifizierten Logos stützt, wird über kurz oder lang von anderen Sozialsystemen aufgesogen." (96/135) Ist also das Ganze letztlich nichts weiter als ein Plädoyer dafür, dass das argumentative Geschehen in der Jurisprudenz auch eine affektive Seite hat?

Die Quaestio crucis dabei ist ja wohl: Was soll im Zweifels- oder im Streitfall den Ausschlag geben, Argumente oder Gefühlsappelle? Und darauf antwortet sie am Ende dann doch mit Formulierungen, die sich kaum anders denn als Bekenntnis zu Tradition und Gefühl als Entscheidungsinstanzen lesen lassen:

„Wenn es sich auch um ein bedauerliches Fortschrittshindernis handeln sollte: die Grammatik des Überzeugens ist älter und sitzt vermutlich tiefer als alle ,postkonventionelle' Theorie. Ob dies allerdings immer von Nachteil sein muss, ist noch nicht erwiesen." (96/135)

Und dann noch:

„Was der Logiker als Unvollkommenheit erlebt, dass sich nämlich in allen längeren Rechtfertigungsketten Risse auftun, die der Richter mit rhetorischen Figuren überbrücken muss, könnte sich also als der gesuchte Weg zu Inhalten erweisen, die als gerecht und tugendhaft empfunden werden. Kriterien für Gerechtigkeit lassen sich vielleicht verlässlicher in verbreiteten Gefühlen als in generalisierbaren Sätzen finden." (ebd.)

\footnotetext{
4 Vgl. Paul Robertshaw, Das richterliche "Summing up“ im Fall Jeffrey Archer, in: Rhetorik Jahrbuch 15 (1996), 96-114, 109.
} 


\section{Kritik}

\subsection{Begriffsanalytische Kritik}

Jede wissenschaftliche Forschung braucht ein Minimum an sprachphilosophischem Gedankengut. Es muss klar sein, dass in den Wissenschaften Wörter nicht einfach aus der Umgangssprache aufgegriffen und verwendet werden können, sondern dass durch Definitionen und Normierungen ausdrücklich Termini gebildet werden müssen: Die Wörter der Umgangssprache haben keine strikten Verwendungsgrenzen, sie können für immer neue und auch immer wieder abgewandelte Sachverhalte eingesetzt werden. Diese "Lebendigkeit” der Sprache hat zur Folge, dass sich kaum jemals ganz klar und eindeutig entscheiden lässt, ob ein aus umgangssprachlichen Wörtern zusammengesetzter Satz als wahr und gültig oder ob er als falsch und ungültig zu beurteilen ist. Für die wissenschaftliche Forschung ist eine solche Beurteilungsmöglichkeit aber unverzichtbar, denn in ihr geht es genau darum, was wahr ist und was nicht. Ohne klare und strikte Definitionen gibt es daher keine wissenschaftliche Erfassung von Sachverhalten. Wir müssen wissen, was wir mit den Wörtern meinen. Wenn man Wörter im Hinbiiick auf ihre - gebenenfalls explizit zu klärende - Bedeutung "Begriffe" nennt, dann ist das Bereitstellen eines theoretischen Apparats für wissenschaftliche Forschung wesentlich "Begriffsbildung". Begriffsbildung ist das systematische Fundament aller Wissenschaft. Obwohl die Wissenschaft sich damit nur eher ausnahmsweise beschäftigt - in der sogenannten Grundlagenforschung - hängt von der Klugheit und Sorgfalt, die auf die Konstruktion des begrifflichen Fundaments gewendet wird, weitgehend $a b$, was für einen Sinn eventuell gefundene Forschungsergebnisse haben.

Sobota möchte mit ihrer Begriffsbildung an Aristoteles anschließen. Dort findet sie die Wörter Logos, Ethos, Pathos vor. Sie greift sie auf und verwendet sie, aber, wie ich zeigen werde, in einem nur vage ausmachbaren Sinn. Die notwendige begriffliche Präzisierung und Schärfung, die kriegt man durch den Verweis auf den klassischen Autor eben nicht geschenkt. Bei Aristoteles sind Logos, Ethos und Pathos nicht so strikt getrennt, als drei separate "Überzeugungsmittel" - wie drei verschiedene Taschen, in denen irgendwelche Inhalte zum Adressaten transportiert würden. ${ }^{5}$ Überhaupt sind dort die Wörter nicht so

\footnotetext{
${ }^{5}$ Sobota verweist $(96 / 118)$ für ihre "Trias“ aus Logos, Ethos und Pathos auf fünf Stellen in der Rhetorik des Aristoteles, an keiner dieser Verweisstellen ist dergleichen zu finden. Für die Hochschätzung der Lebensklugheit bzw. der guten Mitte zwischen den Extremen wird zu Recht die Nikomachische Ethik aufgeboten. Die dabei von Sobota
} 
definiert, wie wir das in unseren heutigen wissenschaftlichen Standards fordern. Das kann man dem Philosophen nicht anlasten. Im vierten vorchristlichen Jahrhundert sah "die Wissenschaft" noch etwas anders aus als heute. Allgemein gesagt kann die Anknüpfung an einen antiken Denker die eine oder andere wichtige Unterscheidung und Einsicht in gewisse Grundverhältnisse vorgeben, aber die eigene begriffliche Anstrengung niemals komplett ersetzen. Ich möchte in diesem ersten Teil der Kritik drei Ausdrücke, die für Sobotas Ansatz zentral sind, auf ihren begrifflichen Gehalt hin überprüfen: Pathos bzw. rhetorische Figur, Logos, bzw. Argument und Überzeugung bzw. Überzeugen.

\subsubsection{Pathos - Die rhetorische Figur}

Gleich zu Beginn ihrer Studie von 92 gibt Sobota zu verstehen, dass es mit der Begriffsbestimmung zur rhetorischen Figur nicht zum Besten steht: „Wie alle Erscheinungen auf dem Gebiet der Künste und praktischen Fertigkeiten entzieht sich die rhetorische Figur einer exakten Definition." (92/232) Nun ist es nicht wahr, dass das für "alle" derartigen Erscheinungen gälte (Gegenbeispiel ist etwa der "goldene Schnitt"), und eigentlich werden in der Wissenschaft auch überhaupt keine "Erscheinungen", sondern allenfalls Wörter oder Termini definiert, aber akzeptieren wir einstweilen, was hier gesagt wird. Es gibt also keine exakte Definition, aber vielleicht doch eine partielle, ein Kriterium oder wenigstens eine umrisshafte Angabe, die sich durchhalten ließe? Sie sei ein "rhetorisches Überzeugungsmittel” (ebd.), wird gesagt. Wüssten wir jetzt, was genau „Überzeugen” sein soll - ich beschäftige mich damit unter (3.1.3.) - dann wären wir weiter. Da wir es nicht wissen, hören wir weiter zu und lassen uns als Nächstes über die Figuren sagen, sie wendeten sich ans "Herz" des Zuhörers (92/232). Dort „bezaubern sie durch ihre Formen: durch Rhythmus, Geometrie, Klang oder Anschaulichkeit" (ebd.). Das ist zweifellos selber sehr anschaulich, sagt aber nicht klipp und klar, was damit gemeint sein soll: Möchte Sobota, wenn sie etwas ,rhetorische Figur' nennt, die Behauptung aufstellen, der Textautor "wende" sich damit ans Herz des Adressaten? Soll das für jeden Einsetzer einer rhetorischen Figur gelten? Für jede Figur? Impliziert die „Wendung” zum Herzen, dass das Herz getroffen wird?

nahe gelegte Ansicht, Aristoteles sei ein Theorieverächter gewesen (ebd.), ist allerdings abenteuerlich. Es ging dem Philosophen darum, dass die Theorie systematisch nicht vor, sondern nach der Praxis kommt. Dann aber ist sie das Größte und Beste, dessen der Mensch überhaupt fähig ist. Über den „bios theoretikos" als die beglückendste Lebensweise gibt das letzte Buch der Nikomachischen Ethik Auskunft. 
Manchmal? In der Regel? Immer? Wie reagiert ein Herz, dem sich zugewendet wurde? Damit, dass es nun „überzeugt" ist? Ist es dafür egal, um welche Sachverhalte es beim Einsatz der Figur ging? Ist Überzeugtsein überhaupt ein Zustand, in dem sich ein Herz befinden kann? Und so weiter. Das sind allerlei offene Fragen, ich vermute, dass Sobota sie kleinkariert findet. Sie hat doch zugegeben, eine "exakte Definition" gebe es nicht. Das kann aber nicht heißen, dass mit dem Wort nach Belieben umgesprungen werden kann. Die Forderung, dass der wissenschaftliche Sprachgebrauch über die situative Beliebigkeit der Alltagssprache hinaus sein muss, ist unverzichtbar.

Schauen wir also nach, was sie an weiteren Bestimmungen gibt: Üblich sei es, die "rhetorische Figur" zu erläutern mit "Abweichung von der normalen Sprechweise", wobei aber der "Begriff der Normalität ... nur relativ bestimmt werden" könne (92/232). Schade, das ist wieder mehr eine Ausflucht als eine Erläuterung: ,Abweichung von der normalen Sprechweise' bedeutet eben nur dann etwas Klares, wenn klar ist, was eine "normale Sprechweise" ist. Das kann, schreibt sie, nur relativ bestimmt werden. Relativ wozu denn? Statt einen Bezugsrahmen anzugeben, stellt sie einen Beispielsatz vor, bei dem die Sache ganz einfach ist, weil der dort gezeigte Unterschied zwischen einer normalen Sprechweise (Sonne scheint) und einer metaphorischen (Sonne lacht, sendet süße Strahlen) offenkundig ist. Wie sich dieses Beispiel auf weitere oder gar alle Figuren übertragen lassen soll, steht in den Sternen. Gerade bei Metaphern ist es in der Regel schwierig zu entscheiden, ob sie als "verbrauchte" bzw. "gesunkene" Metaphern zur normalen Sprechweise gehören (wie z. B. Höflichkeit, Angespanntheit) oder ob dabei wirklich eine Bedeutungsübertragung von einem in einen anderen Bereich stattfindet. Dennoch ließe sich von einer ",normalen Sprechweise" ja reden, solange davon nichts abhängt. Wenn nun aber Normalität als Kriterium für irgendetwas dienen soll (wie hier: für Abwesenheit von rhetorischen Figuren), dann sind wir damit rasch am Ende. Alle Sprachabweichungen bei Kindern oder Nicht-Muttersprachlern (die ja auch interessant oder anmutig sein, die auch das "Herz" anrühren können) wären dann „Figuren".

Diese Überlegungen dürften genügen: Weder Definitionen - exakte ohnehin nicht, aber auch keine laschen - noch Kriterien für "rhetorische Figur" sind hier in Sicht. Geben wir also den Versuch, das selbst zu bestimmen auf und halten uns an die Figurenkanones der traditionellen Rhetoriken? Aber da lässt sich sehr vieles haben und weniges mit Gründen ausschließen. Sobota erwähnt selbst die „unübersehbare Menge der klassischen Figuren" (92/233). Tendenziell ist eben jedes irgendwie differenzierte Sprachvorkommnis Figur. Die traditionelle 
Rhetorik, daran muss erinnert werden, ist ja auch gar nicht primär spezialisiert auf die Aufgabe, zu „überzeugen”, sondern darauf, ein Bewusstsein von den Möglichkeiten sprachlicher Differenzierungen zu erzeugen, das dem allgemeinen Ziel, den Zuhörer zu erreichen, ihm eine Botschaft - und zwar ohne Unterstützung durch optische Medien - zu vermitteln, dienlich ist. Figurenreiche Sprache ist also zunächst einmal nichts weiter als elaborierte, differenzierte Sprache. ${ }^{6}$

Dieses Bedenken muss sich nun gegen Sobota kehren. Sie hat im $\mathrm{Zu}-$ sammenhang mit "rhetorischer Figur", von einem "Überzeugungsmittel" gesprochen. Stellen wir zurück, dass wir noch zu untersuchen haben, was überhaupt ,Überzeugen' heißen soll, so ist es doch offenbar irgendein Effekt, den die Adressaten verspüren sollten. Das bedeutet aber, hier sind weitreichende Beweislasten zu schultern. Wenn etwas ,Figur' genannt wird, dann müsste es eine solche Überzeugungswirkung auf den Adressaten haben oder wenigstens müsste beim Autor die Intention unterstellt werden, eine solche zu erreichen. Dass sich das so verhält, lässt sich aber nicht pauschal fordern, sondern müsste für jedes einzelne Ding, das ,Figur' genannt werden soll, nachgewiesen werden. Davon ist Sobota weit entfernt, weder tut sie das, noch ist zu merken, dass sie eine entsprechende Verpflichtung akzeptieren würde. Was heißt also bei ihr "rhetorische Figur"? Ganz einfach: Das, was sie so nennen will. ,Rhetorische Figur' ist die Bezeichnung für ein Vorkommnis aus ihrer 37er-Liste. Was ist das Gemeinsame dabei? Wir wissen es nicht, wir wissen nicht einmal, ob es überhaupt etwas Gemeinsames gibt. Jedenfalls ist die Liste so heterogen, dass Unterschiede gerade hinsichtlich der Qualität des Gefühlsappells - die für Sobotas Argumentation die entscheidende ist - ins Auge fallen. Was in aller Welt sollen Parenthese, Periphrase und Zitat mit Metapher, Emphase, Hyperbole usw. gemeinsam haben? Einzig und allein die Tatsache, dass sie in Rhetoriklehrbüchern seit unvordenklichen Zeiten aufgeführt und immer wieder abgeschrieben worden sind!

Ein genaueres Hinsehen entdeckt bei vielen Einträgen dieser 37erListe die schiere Willkür: Wie wirkt Ironie - eher vereinnahmend oder eher abstoßend? Lässt sich das wirklich allgemein beantworten? Ist das nicht davon abhängig, wer oder was ironisiert wird, davon, ob die Sache dem Auditorium egal ist, von ihm abgelehnt wird oder ihm etwa

\footnotetext{
${ }^{6}$ Diese Feststellung reicht, um einen Befund, den Sobota für ihre Hypothese ins Feld führt, zu erklären: dass die durchschnittliche Figurendichte der BVerfG-Urteile in der Höhe zwischen den Feuilleton-Artikeln in Lokalblättern und denen in überregionalen Zeitungen liege (92/233). Figurenreichtum ist ein Indikator für den Bildungsstand, der ist bei den überregionalen Journalisten höher als bei den regionalen. Die BVerfG-Richter aber sind gebildeter als die Lokalredakteure und sie schreiben nüchterner und sachlicher als die Feuilletonisten überregionaler Blätter.
} 
besonders am Herzen liegt? Sogar "vielsagendes Schweigen" soll eine Pathos-Figur sein. Werden Gefühle dadurch angesprochen, dass vielsagend geschwiegen wird? Wieso nicht eher Gedanken? Wieso ist "raffiniertes Pathos" überhaupt "Pathos"? (Vgl. das oben zitierte Beispiel mit der Krawatte: „Im Prinzip ist diese Krawatte nicht hässlich, sie passt nur nicht zu den roten Streifen in deinem Anzug." Dies ist ein anständiges ästhetisches Argument; und wenn Kriterien für Farbharmonie etabliert sind, dann kann es sogar auf Gültigkeit überprüft werden.) „Pathos der Sachlichkeit” (96/124), das ist eine Aus- und Überdehnung der für "Pathos" vorgetragenen Charakterisierungen (anschaulich, emotional, bezaubernd) ins genaue Gegenteil. Weiter: Wieso wird die Restrictio überhaupt als rhetorische Figur geführt? Eine ZwarAber-Konstruktion wendet sich ganz bestimmt eher an den Verstand als ans Gefühl. Dennoch kann sie eine suggestive Vereinnahmung anbahnen, aber nur dann, wenn die argumentativen Gehalte des Zwarund die des Aber-Teils eigentlich gleichwertig sind. Beispiel: Man sollte sich einen Hund halten, zwar verursacht er Kosten und erschwert das Reisen, aber er bewacht das Haus und ist der beste Freund des Menschen. So redet, wer seine hundeskeptische Frau für den Hund einnehmen möchte. Und wer seine hundefreundliche Frau vom Hund abbringen will, der dreht die Sache um: Besser keinen Hund! Zwar könnte er das Haus bewachen und sich als guter Freund bewähren, aber er ist teuer und wir können nie mehr verreisen. Derartige Manöver sind aber, wie gesagt, nur möglich, wenn Zwar-Teil und Aber-Teil gleichwertig sind, wenn also die für den Sachverhalt verfügbare theoretische Basis keine Überlegenheit für einen der beiden hergibt. Ist das anders und bringt $z$. B. der Aber-Teil eine wirkliche Widerlegung oder Integration des Zwar-Teils, dann ist eine solche Konstruktion ein gutes Argument, das einen Einwand aufgreift und ausräumt. Beispiel: Zwar hat Sobota im Grunde etwas Richtiges erkannt, aber sie verfolgt es mit ganz untauglichen Mitteln (für die Ausführung dieses Beispiels vgl. die Schlusspassage dieses Aufsatzes).

Fazit: Sobotas Versuche, einen Begriff der "rhetorischen Figur" zu bilden, sind partiell informativ, anschaulich und suggestiv, scheitern aber im Hinblick auf das Bilden einer stabilen semantischen Einheit. Thre Erläuterungen wie die Wendung zum Herzen, das Bezaubern, das Überzeugen, das sind lauter Versicherungen, die teils mehr, teils weniger, teils überhaupt nicht plausibel sind und die sie als Charakterisierungen ihrer "Figuren" ungeprüft hinstellt. De facto besteht ihre Definition in einer Aufzählung von 37 Text-Vorkommnistypen, die so heterogen sind, dass die Zusammenstellung beliebig und willkürlich erscheint. 


\subsubsection{Logos - Das Argument}

Die rhetorische Figur entzog sich, wie gesehen, einer "exakten Definition", beim Argument taucht die Frage danach gar nicht erst auf. Wie oben referiert, wird bei Sobota "der Begriff ,Argument' ... sehr weit verstanden" (96/120). Es sollen nämlich "Aussagen oder Andeutungen" (ebd.) darunter fallen, die sich als ", $\mathrm{X}$ weil $\mathrm{X}$ " lesen lassen, wobei $X$ die "weitere Begrifflichkeit", etwa eine Regel oder ein allgemeiner Wert sein soll. Die Autorin denkt bei Argumenten offenbar an deduktive Schlüsse aus allgemeinen Sätzen, wie sie in Rechts- oder Moralnormen auftreten, auf Einzelsätze, die sich auf konkrete Situationen beziehen. Zur näheren Bestimmung unterscheidet sie bei den allgemeinen Prämissen, aus denen geschlossen werden kann, drei Arten, nämlich „Kodifikationsargumente", deren Prämissen Normen des positiven Rechts sind, "Rechtsgrundsätze", deren Prämissen offenbar Prinzipien für die Ausbildung des positiven Rechts sind, und "Alltagsargumente", die vorliegen, wenn aus generellen Sätzen des Alltagslebens wie Erfahrungen, Sprichwörtern usw. geschlossen wird. Sie hat noch eine vierte Gruppe, nämlich die „Folgerungen”, die sie als Argumente zählt, obwohl es keine seien. Es müsse dafür nur „ein sicherer Hinweis auf die Prätention einer Prämisse" (96/120) vorliegen. Wie ist das zu verstehen? Wenn an irgendeiner Stelle im Text der Eindruck entsteht, der jeweilige Satz solle nicht für sich selbst stehen, sondern sei erschlossen aus einer Prämisse, die auch implizit sein kann - es muss nur "ein sicherer Hinweis" darauf vorliegen - dann ist er eine "Folgerung". Und dann wird er gezählt, d. h. es wird ein Punkt für "Logos”, bzw. „Argument" gegeben.

Ich fasse zusammen: Argument heißt hier so viel wie "Bestandteil eines logischen Schlusses". Der logische Schluss muss nicht ausformuliert sein, es reichen „Andeutungen". Sind Sätze oder genügend starke Hinweise da, dann zählen die Prämissen als Argumente und die Schlusssätze ebenso. Statt von Prämissen spricht Sobota auch von "Begründungen", wie üblich, ohne genau zu sagen, was sie darunter versteht. Ist der allgemeine Satz, aus dem auf den Einzelsatz geschlossen wird, die "Begründung"? Oder ist es der allgemeine Satz als Oberprämisse plus der konkretisierte Bedingungsteil dieses allgemeinen Satzes als Unterprämisse? Oder soll die "Begründung" das ganze logische Antezedens sein, das für den Schluss nötig ist? Dann würden meistens noch zahlreiche implizite Prämissen, die als gegeben vorausgesetzt werden, insbesondere stets das logische Schlussschema als weitere Oberprämisse dazu gehören. Vermutlich hat Sobota solche Möglichkeiten vor Augen, wenn sie schreibt: "Sind zwischen Behauptung und Be- 
gründung offenbar noch weitere Prämissen eingeschaltet, zählen diese nur, wenn sie im Text Anklang gefunden haben" (ebd.). Wie oben bei den "Andeutungen" ist hier mit dem "im Text Anklang finden" nochmals ein breiter Spielraum für verschiedene Lesarten des Textes und damit für das Erfassen von "Argumenten" offen.

Was ist von diesem Argument-Begriff zu halten? Zunächst einmal ist es ganz abwegig zu glauben, der Begriff sei „sehr weit verstanden”. Er ist im Gegenteil sehr eng. Er verpflichtet den Leser auf den logischen Schluss, und sogar hier noch auf einen Teilbereich, den nämlich, der das syllogistische Ableiten von generellen auf singuläre Sätze betrifft. Dass schon die klassische Logik (seit der Stoa) viel reicher ist und dass in der gewöhnlichen argumentativen Rede, auch der Jurisprudenz, weit mehr logische Schlüsse vorkommen, das scheint Sobota noch nicht beobachtet zu haben. ${ }^{7}$ So elementare Dinge wie der Schluss von einer Konjunktion auf einen der Teilsätze oder von einer Alternative plus der Negation des einen Teilsatzes auf die Affirmation des anderen Teilsatzes würden schon durch ihr Raster fallen. Ganz abgesehen von all dem, was - außerhalb des formallogischen Schlusses - sonst noch mit Sinn als "Argument" bezeichnet wird: Generalisierungen, Analogien, Dammbruchargumente usw. Schließlich gibt es die große Sparte von Text-Vorkommnissen, die überhaupt keine Begründungen (oder Teile von solchen) sind und die dennoch zu Recht "Argumente" heißen, nämlich Einwände gegen die These oder Gegenargumente. (Das kann z. B. so aussehen: ,das Gericht hatte sich mit folgendem Bedenken auseinanderzusetzen ...' oder: ,Hier ist der Einwand gebracht worden, dass ...') Wie sollte so etwas mit Sobotas Erläuterungen fassbar sein? Es ist weder eine Prämisse, noch die Prätention einer Prämisse, noch eine Folgerung. Was ist es denn? Es ist das argumentierende Aufgreifen eines Versuchs, die These oder ein schon genanntes Argument als ungültig zu erweisen.

Bis hierhin habe ich lauter Einreden und Gesichtspunkte gebracht, die sich immerhin auf Sobotas Ansatz, also "Argumente" an bestimmten Text-Vorkommnissen festzumachen, einlassen. Es ist aber der Ansatz selbst ganz unzweckmäßig und er muss zu willkürlichen und phantastischen Resultaten führen. Argumente stehen in einem Text nicht wie Gänseblumen in der Wiese herum, sondern Argumente sind pragmatische Strukturen, die einen Text organisieren. Argumente gibt es, wenn Thesen (Behauptungen) aufgestellt sind und als geltend ausgewiesen

\footnotetext{
7 Vgl. ULRICH KLUG, Juristische Logik. Berlin 1951. Mit diesem Buch, inzwischen in vierter Auflage erscheinend, müsste eigentlich für Juristen seit einem halben Jahrhundert das Klischee von einer juristischen Logik, die sich in der Ableitung aus generellen Normen erschöpfte, veraltet sein.
} 
werden sollen. Argumente sind dann Produkte der sprachlichen Handlungen, die unternommen werden, um diesen Geltungsanspruch einzulösen. Für das Ausführen solcher sprachlicher Handlungen hat ein Autor keine regulären Vorschriften hinsichtlich der Wortwahl oder der Länge des Textes oder seiner Gliederung in Teile. Hat er eine logische Schulung durchlaufen, wird er meistens bestrebt sein, Sätze zu produzieren, die in irgendeinem Sinne als „implizierende " Prämissen für die Behauptung als Konklusion fungieren können. Aber ob er pro Prämisse einen Satz oder zwanzig braucht, das steht in seinem Belieben. Ebenso steht weitgehend in seinem Belieben, wie viele Prämissen er für seine Argumentation braucht. Das wird davon abhängen, was er selber für selbstverständlich hält und was er bei seinen mutmaßlichen Adressaten als gewusst und verstanden unterstellen kann. Das alles ist aber so offen, dass es dazu (richtigerweise) keine regelhaften Festsetzungen gibt. Ferner ist es möglich, dass er den Übergang, den er sich zwischen der Prämissengruppe und der These bzw. Konklusion denkt, durch spezielle Wörter wie ,weil', ,daher', ,deshalb', ,also' usw. bezeichnet und dadurch verbal kenntlich macht. Doch auch dies ist keineswegs notwendig.

Ein realistischer und sinnvoller Argumentationsbegriff kann nicht in der Syntax oder der Semantik der Sprache, sondern muss in der Pragmatik des Redens ansetzen. Beim Argumentieren wird mit der Sprache etwas getan oder wenigstens zu tun versucht. Das hat zur Konsequenz, dass keine an äußerlichen Textmerkmalen identifizierbare Einheit für „ein Argument" da ist. Und das wiederum hat zur Konsequenz, dass es hier nichts zu zählen gibt, jedenfalls nichts, was eine sachliche Aussagekraft hätte. Ich werde das unter der wissenschaftstheoretischen Kritik noch genauer zeigen.

Das allerwichtigste aber, was bei der Bestimmung eines Argumentbegriffs zu berücksichtigen ist, das ist der Geltungsbezug. Argumente sind, wie gesagt, Versuche, eine These als gültig (wahr) auszuweisen. $\mathrm{Da}$ die These nicht zum Wissen gehört, sondern das Wissen überschreitet (überschreitet sie es nicht, braucht nicht argumentiert zu werden, dann lässt sich im Lehrbuch nachschlagen), tragen die Argumente die Last des Ausweises. Diese Last impliziert, dass sie sich gegebenenfalls selbst - als Subthesen - auf ihre Geltung hin ausweisen müssen. Argumente haben einen intrinsischen Geltungsbezug. Wer eine Argumentation analysieren und nicht danach fragen will, wieweit sie als Ganzes und in ihren einzelnen Argumenten die These als gültig oder ungültig ausweist und ob die Argumente selbst gültig oder ungültig sind, der braucht sich nicht zu wundern, wenn er wenig Greifbares und Stabiles zutage fördert. Er hat das Telos des argumentierenden Redens 
glatt ignoriert. Ein Text ist nicht dadurch rational, dass Sätze verschiedener Formen (generelle, partikulare, singuläre) darin vorkommen und Wörter, die für logische Strukturen symptomatisch sein können, sondern ein Text ist dadurch rational, dass er einen Sachverhalt in einer Kette von Argumenten durchsichtig und richtig darstellt. Dazu müssen diese Argumente aber stimmen oder wenigstens sinnvollerweise als stimmig gemeint sein können. Ein Text, der Scheinargumente und primitive Klischees in einer Prämissen-Konklusions-Struktur präsentiert, ist kein rationaler Text. Wenn Sobota glaubt, die Rationalität von juristischen Urteilen darin zu erfassen, wie sich die von ihr "Argumente" genannten Text-Vorkommnisse zu den rhetorischen Figuren verhalten ohne nachzuschauen, ob ihre "Argumente" überhaupt gültig sind, dann zeugt das von einer sehr grundlegenden Konfusion.

Dieser Gesichtspunkt des fehlenden Geltungsbezugs betrifft auch die unter dem Titel "Ethos" abgetrennten Traditionsargumente. Sowohl Autoritäts- als auch Textargumente und Quellenangaben können ja schlicht falsch sein. Bei Zitaten und Quellenangaben ist die Richtigkeit meistens durch einfachen Vergleich überprüfbar. Bei Autoritätsargumenten ist im Zweifelsfall eine Beurteilung des fraglichen Sachverhalts (z. B. ob es dazu überhaupt Expertenwissen gibt) sowie eine Bewertung der Autorität (hinsichtlich Kompetenz und Zuständigkeit für den fraglichen Sachverhalt) nötig, um sie rational akzeptieren oder zurückweisen zu können. Das bloße Faktum des Vorkommens von Autoritätsverweisen, Zitaten oder Quellenangaben spricht noch nicht, wie Sobota glauben machen möchte, für "professionelle Gewissenhaftigkeit, Kontinuitätsbestreben und Achtung vor dem Hergebrachten" (96/121). Das ist vielmehr nur dann der Fall, wenn diese "Ethos-Argumente" auch gültig sind.

Fazit: Sobotas Argumentationsbegriff ist viel zu eng, um das argumentative Geschehen zu erfassen, er ist zu vage, um verfahrensmäßig anwendbar zu sein, und er ist rein äußerlich, hat keine inhaltliche Substanz, weil ihm der Geltungsbezug fehlt.

\subsubsection{Das Überzeugen}

Der dritte Ausdruck, den ich auf seinen begrifflichen Gehalt prüfen und kritisieren möchte, ist das Überzeugen. Hier ist der Befund in Sobotas Schriften - soweit von mir studiert - einfach: Sie hat gar keinen Begriff vom Überzeugen, sie verwendet das Wort, Überzeugen' als sei dabei nichts weiter zu verstehen oder misszuverstehen. Es wird unterstellt, dass die BVerfG-Urteile ,überzeugen', es wird nicht unterschieden, ob sie das wollen, sollen oder ob sie das vielleicht sogar tun und 
falls ja, wen sie denn überzeugen. Man könnte sich die Sache leicht machen und verstehen, Texte „überzeugten", insofern sie mit der Intention verfasst worden sind, dass die Rezipienten zustimmen mögen. Dann wäre aber z.B. die ganze kommerzielle und politische Werbung, und zwar mit all ihren widerwärtigen Ausuferungen, „überzeugend".

Sicherlich kann sich Sobota an einen Sprachgebrauch vom „persuasiven" Reden anschließen. Der Ausdruck ist ein Derivat des lateinischen ,persuadere' und das bedeutete bekanntlich (genauso wie vorher schon das griechische ,peithein') so etwas wie ,Glauben machendes Reden'. Aber es waren schon die Philosophen der Antike, ganz besonders der von Sobota bemühte Aristoteles, die die Notwendigkeit erkannten, hier eine Differenzierung einzuziehen. Es muss unterschieden werden, ob bei einer Rede nur irgendwelche oberflächliche Zustimmung oder ob ein reflektiertes Einverständnis mit einer These erreicht wird bzw. ob darauf abgestellt wird. Dass sich mit Fehlschlüssen und rhetorischen Kniffen Zustimmung erzeugen lässt, dass das aber dann ein beklagenswerter Zustand ist, weil es eine schlechte, irregeführte, nicht tragfähige Zustimmung ist, das hat z. B. Aristoteles bewogen, die Manöver der sophistischen Rhetorik zu analysieren und aufzuklären. ${ }^{8}$ Das Ergebnis war die erste Sammlung von Fallazien (Argumentationsfehler), die wir besitzen. ${ }^{9}$ In der Folge, in der mittelalterlichen Dialektik, ist immer weiter zwischen einer durch Trick und Irrtum hergestellten Scheinüberzeugung und einer der geltungsbezogenen Reflexion standhaltenden wirklichen Überzeugung unterschieden worden. Im Deutschen ist seit Kant dafür der Unterschied zwischen, Überreden' und ,Überzeugen' eingebürgert. ${ }^{10}$ Diesen Unterschied einsetzend können wir sagen, dass die Botschaften der Werbung in der Regel überreden wollen und das (leider) oft auch tun. In seriöser Argumentation, also insbesondere in der Jurisprudenz und in der Wissenschaft, wird aber nicht zu überreden, sondern zu überzeugen versucht.

Für einen anspruchsvollen Begriff von ,Überzeugen' ist, wie für den Argumentationsbegriff, wieder der Geltungsbezug nötig. Ein Text ist überzeugend, wenn ich als Rezipient nach Prüfung und Überdenken zu dem Ergebnis komme, dass die in ihm vorgetragene Botschaft (Behauptung, These) richtig bzw. gültig ist. Dieses Ergebnis gehört damit dann zu meinen Überzeugungen. Die müssen nicht für alle Zeiten stehen

\footnotetext{
${ }^{8}$ Vgl. ARISTOTELES, Sophistische Widerlegungen (Organon VI). Übersetzt und mit Anmerkungen versehen von EUGEN ROLFES. Hamburg 1968

${ }^{9}$ Im Corpus Aristotelicum gibt es mehrere Listen von Fallazien, bzw. fehlerträchtigen Topoi, die ausführlichste im zweiten Buch der Rhetorik.

${ }^{10} \mathrm{Vgl}$. IMmanuel Kant, Vom Meinen, Wissen und Glauben, in: Ders., Kritik der reinen Vernunft. Riga 1781, A 820/ B 848 ff.
} 
können, aber mein gegenwärtiger Stand von Information und Reflexionskompetenz, der drückt sich darin aus. Ich denke, dass wir alle (auch Sobota) glauben oder mindestens hoffen, die Urteile des BVerfG seien in diesem anspruchsvollen Sinne "überzeugend". Wenn das soweit richtig ist, hat es zur Konsequenz, dass sich Argumente und rhetorische Figuren überhaupt nicht unter dem Oberbegriff "Überzeugungsmittel" zusammenfinden können. Wer überzeugen möchte, hat Argumente zu bringen. Wer prüfen will, ob ein Text überzeugend ist, hat die Argumente zu prüfen. Die rhetorischen Figuren können im besten Fall beim Prozess der Prüfung helfen, indem sie das Material beleben, verschönern, schmücken. Für den Unterschied, ob der Text überzeugt oder nur überredet, tragen sie nichts bei.

\subsection{Wissenschaftstheoretische Kritik}

\subsubsection{Basisfeststellungen zur empirischen Forschung in den Sozial- und Textwissenschaften}

Sobota behauptet, dass sie ihre Resultate zu der "messbaren Balance" zwischen Logos, Ethos, Pathos, in der sich Rechtstexte und besonders die BVerfG-Urteile befänden, und zur Dominanz von Pathos gegenüber Logos an den entscheidenden Stellen nicht durch "weiche" Verfahren hermeneutischer und interpretierender Textanalyse, sondern durch harte messende Empirie gewonnen habe. Diese Behauptung ist nach der Begriffsbildungskritik fraglich geworden. Sie soll jetzt auch noch wissenschaftstheoretisch beleuchtet werden. Dabei geht es hauptsächlich um die Frage, ob Sobotas Rechtstextanalyse wirklich empirisch ist. Vielleicht ist es ratsam, mit einigen Vergegenwärtigungen und Feststellungen zur Wissenschaftstheorie der Sozial- und Textwissenschaften zu beginnen.

Empirische Forschung ist methodische und intersubjektiv nachvollziehbare Erfassung der äußeren Wirklichkeit. Empirisch erforschen lässt sich z. B., wie sich das Klima in einer bewaldeten Region ändert, wenn der Wald abgeholzt wird; oder wie sich das Wahlverhalten der Anhängerschaft einer bestimmten Partei ändert, wenn bei dieser Partei ein Spendenskandal aufgedeckt wird. Für die Forschung ist es nötig, dass die Frage „Wie ist die Wirklichkeit?” spezifisch eingeschränkt wird, so dass die interessierenden Aspekte klar hervortreten. Dazu wird das Vorverständnis der betroffenen Sachverhalte aktiviert, es werden die relevanten Unterschiede und Verhältnisse begrifflich artikuliert und diese Begriffe sodann so "operationalisiert", dass sich damit „Fragen” stellen lassen, die die Wirklichkeit „beantworten” kann. 
Für die präzise Fragestellung wird im Allgemeinen eine Hypothese gebildet, denn die "Antworten" der Wirklichkeit sind nicht gerade prosaisch, sie besagen nur ja/nein oder soundso groß. Begriffliche Artikulation, die Operationalisierung der Begriffe in Parametern, die Bildung einer Hypothese bzw. eines Hypothesengeflechts stellen zusammen einen "theoretischen Apparat" dar, der in einem "Messgerät" gipfelt, mit dem dann Daten erhoben werden können. Bei experimenteller bzw. intervenierender Forschung muss der Apparat reicher sein, er muss auch Sätze über gesetzmäßige Verläufe im Gegenstand enthalten, bei rein rezeptiver Forschung spielt das keine Rolle. Die erhobenen Daten bilden zusammen die "Datenbasis", die dann in Beziehung zur Hypothese gesetzt werden kann. Im einfachen Fall erlaubt sie, die Hypothese auf Gültigkeit (relativ zur Datenbasis) zu beurteilen. Beim Klimaforschungsprojekt hätten wir Begriffe zu bilden, die den IstZustand des Klimas darstellen, und zwar mit veränderlichen Größen, sodann Begriffe, die die "Abholzung des Waldes" erfahrbar machen. Beim Wahlforschungsprojekt müsste "Wahlverhalten" in veränderlichen Größen und "Spendenskandal" in eindeutigen, gegebenenfalls quantitativen Parametern erfasst werden.

Die Bedeutung dieser theoretischen Vorarbeiten zur Herstellung des Messgeräts ist buchstäblich "fundamental". Wenn sich hier Fehler einschleichen, stellen die erhobenen Daten nicht die Wirklichkeit, sondern irgendetwas anderes innerhalb der Relation des Forschers zur Wirklichkeit dar. Um den Wirklichkeitskontakt zu sichern, ist es eine Grundforderung, dass das Messgerät stabil ist, d. h. dass es unter Standardbedingungen unverändert bleibt, und damit Messungen erlaubt, die vom jeweiligen Forscher unabhängig sind.

In den Sozialwissenschaften sind die Messgeräte im Allgemeinen Tests oder Fragebögen. Für deren Stabilität sind drei Standardforderungen etabliert: Objektivität, Reliabilität und Validität. Objektivität bedeutet, dass das Funktionieren des Messverfahrens unabhängig ist vom jeweiligen Forscher, seinen Vorlieben, seiner Lust, Laune usw., so dass seine Resultate prinzipiell von jedem anderen Forscher überprüft werden können. Reliabilität bedeutet, dass die Resultate reproduzierbar sind, also bei Wiederholungen des Verfahrens sich wieder einstellen. Validität bedeutet, dass die Resultate des Verfahrens mit dem, was nach dem Vorverständnis erwartbar war, übereinstimmen. Die drei Forderungen sind hierarchisch gestaffelt. Objektivität ist die grundlegende. Ein Verfahren, das nicht objektiv ist, kann nicht reliabel, eines, das nicht reliabel ist, kann nicht valide sein. Die Unterschiede dieser Forderungen und ihre Rangfolge werden sich gerade für die Einschätzung von Sobotas Ergebnissen als aufschlussreich erweisen. 


\subsection{2. Überprüfung des Analyseverfahrens}

Im Folgenden werde ich den Versuch machen, das Analyseverfahren in einigen exponierten Details nachzuvollziehen. Wenn das Verfahren, wie unterstellt, objektiv ist, müsste es gelingen, die Beispiele, die Sobota in ihren Studien für die Identifikation von Pathos-Stilmitteln und von Logos-, bzw. Ethos-Argumenten gibt, anhand ihrer Definitionen und Beschreibungen als korrekt oder mindestens willkürfrei machbar einzusehen. Diese Identifikationen generieren die Datenbasis für die Auszählergebnisse und die Gegenüberstellung von rhetorischem und argumentativem Gehalt in den synoptischen Kurvendiagrammen. Die Datenbasis müsste intersubjektiv verifizierbar sein, wenn die resultierenden Diagramme als Ergebnisse wissenschaftlicher Forschung akzeptabel sein und wenn deren rechtstheoretische Interpretationen als durch sie gestützt angesehen werden sollen.

Bei den Pathos-Vorkommnissen beschäftige ich mich ausführlich mit der Metapher. Das ist die Figur, die insofern eine exponierte Stellung hat, als sie Sobotas Charakterisierung vom gefühlsappellativen Bezaubern und anschaulichen Beleben des Textes noch am ehesten erfüllt. Für evtl. „bildhafte Qualitäten" eines Textes sind hauptsächlich Metaphern und Analogien verantwortlich, und weil Bilder oft direkter wirken als Gedanken, können Metaphern, jedenfalls wenn sie ungewöhnlich und eindrucksvoll sind, durchaus eine „prima facie“-Eingängigkeit eines Textes besorgen.

Das Ergebnis meines Versuchs, Metaphern-Identifikationen willkürfrei nachzuvollziehen, ist, um das gleich zu sagen, ziemlich entmutigend. Dass das Auffinden und Auszählen von Metaphern durch "Rater" ein objektives Verfahren sein soll, das muss entschieden bezweifelt werden. Damit erweist sich dann eine, wie gesagt, exponierte Figur aus der Liste der "rhetorischen Stilmittel" als wenig tauglich, Sobotas Ziele zu fördern.

Was sodann die Überprüfung der Analyse auf Argument-Vorkommnisse betrifft, so ist die Aufgabe des Nachprüfenden einerseits leichter, andererseits viel schwieriger als bei der Pathos-Analyse. Leichter ist sie, weil sich die von Sobota in ihren Studien erwähnten Logos(und Ethos-)Vorkommnisse einfach daraufhin befragen lassen, ob sie nach den gegebenen Beschreibungen für "Argumente" willkürfrei identifizierbar sind. Schwieriger ist die Aufgabe, weil ich zunächst nicht weiß, was in den Texten, die analysiert werden und aus denen ich als Leser nur Zitate zu sehen bekomme, tatsächlich an Argumentation stattfindet und welche Funktion also die von Sobota identifizierten "Argumente" für das argumentative Geschehen im Text tatsächlich ha- 
ben. Um dazu wenigstens einigermaßen urteilsfähig zu werden, habe ich mir an zwei Beispielurteilen, auf die in der 96er-Studie Bezug genommen wird ("Senatsbesetzung" und "Solange $\mathrm{II}^{\prime)}$, die zentralen Passagen angeschaut, sie auf die Argumentation hin analysiert. Vor diesem Hintergrund beurteile ich die Angaben, die Sobota macht. Auch hier ist es so, dass von einer eindeutigen oder gar zwingenden Identifikation der Vorkommnisse keine Rede sein kann.

Bevor ich mit der Detailüberprüfung beginne, möchte ich wenigstens kurz andeuten, was Sobota tun müsste, um aus den überlieferten rhetorischen Kanones eine Sammlung von Figuren herzustellen, über deren "Wirkung" sie in einer einlösbaren Weise reden könnte. Zunächst müssten für mögliche "Wirkungen" von sprachlichen Ausdrücken oder Redefiguren differenzierende Termini gebildet werden. Es könnte z. B. zwischen „einnehmenden“ und "abstoßenden" Wirkungen unterschieden werden, evtl. mit graduellen Abstufungen. Dann müssten Standardtexte erstellt werden, in denen ein bestimmter Inhalt einerseits mithilfe der $\mathrm{zu}$ testenden besonderen Redefigur und andererseits ohne sie ausgedrückt ist. Hierbei wird die Frage, wie verschieden formulierte Texte inhaltsgleich sein können, beantwortet werden müssen und es werden sinnvolle Kriterien für eine "normale" bzw. "neutrale” Ausdrucksweise zu finden sein. Dann müsste die "Wirkung" des neutralen mit der des figurenhaltigen Textes verglichen werden. Ergeben sich signifikante Unterschiede bei den Wirkungen der Texte, die eine besondere Redefigur enthalten, dann hätte man einen Kandidaten für eine "rhetorische Figur" in dem Sinne, den Sobota für ihre 37er-Liste unterstellt hat. Es müssten dann noch Tests zur Stabilisierung und Erhärtung des Wissens über die Wirkung der fraglichen Figur folgen. Solch eine Prozedur wäre für jede Figur einzeln durchzuführen. Sie wäre gegebenenfalls $z u$ ergänzen um Untersuchungen, wie Kombinationen von Figuren wirken. Verändert sich z. B. die Wirkung, wenn eine Metapher wiederholt wird? Schwächt sie sich ab, verstärkt sie sich? Ich kann das hier nur skizzieren, hoffe aber einen Eindruck zu vermitteln von dem Aufwand, den Sobota treiben müsste, wenn sie es mit ihren Behauptungen über den Charakter ihrer rhetorischen Figuren ernst meinte.

Ich will übrigens nicht verhehlen, dass es mich wundern würde, wenn da überhaupt signifikante Ergebnisse zustande kämen. Ich vermute nämlich, dass sich die gesamte überkommene Textschmuckrhetorik mehr und mehr einfach erledigt, seit wir alle dem Trommelfeuer einer Werberhetorik ausgesetzt sind, die ständig und in aller Öffentlichkeit mit immer neuen überraschenden Sprachabweichungen spielt. Möglicherweise wird doch in Zukunft der schlicht formulierte, 
ganz und gar unprätentiöse Text die größte Aussicht auf ein geneigtes Gehör bekommen. Soviel zu diesem Punkt. Und nun möchte ich mit der Untersuchung der Figuren-Identifikation am Beispiel der Metapher beginnen.

Im Anhang zur 95er-Publikation, in dem die Figuren beschrieben sind, heißt es über die Metapher:

„Übertragung, bildlicher Ausdruck: Sie verloren sich im Dschungel der Großstadt; sie warf ihm einen glühenden Blick $\mathrm{zu}$; seine Miene versteinerte sich. In der Stadt ist kein Dschungel, im Blick keine Glut, im Gesicht kein Stein: Alle drei Ausdrücke beziehen sich nicht auf das, was sie üblicherweise bezeichnen, sondern werden in einem übertragenen Sinn gebraucht. Zwischen dem Gesagten (versteinern) und dem eigentlich Gemeinten (reglos werden, erstarren, Ablehnung signalisieren) besteht keine tatsächliche, sondern nur eine eingebildete ,geistige' Ähnlichkeit." (95/205)

Offensichtlich ist diese Erläuterung bemüht, den Unterschied zwischen der übertragenden und der direkten Bedeutung herauszuarbeiten und dafür Beispiele zu geben. Wir dürfen erwarten, dass die Beispiele, da sie als Vorbilder fungieren sollen, nach Auffassung der Autoren wirklich so deutlich wie nur möglich sind. Für den deutschsprachigen Leser bedeutet das z. B. die erstaunliche Eröffnung, der Ausdruck, die Miene versteinert sich, meine "eigentlich", sie werde reglos oder erstarre, signalisiere Ablehnung. Sieht Sobota nicht, wie vertrackt die Unterscheidung zwischen dem Gesagten und dem "eigentlich Gemeinten" schon an diesem Beispiel ist? Wieso ist denn ,reglos werden' das, was mit dem Reden vom Versteinern eines Gesichts "eigentlich" gemeint ist? Wenn ich von einem Hund oder einem Vorhang gesagt habe, er "rege" sich, dann kann ich, wenn das aufhört, sagen, jetzt sei er „reglos”. Aber wieso ist an einer Miene, die sich versteinert, das „Eigentliche”, dass sie sich nicht mehr regt? Ob Sobota noch nie eine sich versteinernde Miene gesehen hat? Sie „signalisiert Ablehnung"? Ist es nicht prätentiös, zu behaupten, das sei die "eigentliche” Bedeutung des Versteinerns? Kann es nicht Schrecken sein, oder blitzartige Erkenntnis? Und wieso ist denn ,Signalisieren' selber keine Metapher? Ist es irgendwie natürlich oder notwendig, eine sich versteinernde Miene als ein "Signal" aufzufassen?

Allgemeiner gesagt, gebraucht Sobota hier den Unterschied zwischen einer normalen und einer besonderen, übertragenden Redeweise. Solch ein Unterschied lässt sich in einer semantisch stabilen, z. B. einer formalen Sprache machen. In der natürlichen Sprache ist er nur unter großen Schwierigkeiten zu haben. Die natürliche Sprache bewegt sich, ändert sich, sie lebt. Und dieses Leben, das bewerkstelligt sie zu einem erheblichen Teil dadurch, dass sie Metaphern normalisiert. Ich habe das ja oben schon angesprochen. „Verbrauchte" oder "gesunkene" Me- 
taphern heißen solche Ausdrücke, die ursprünglich übertragend waren, dann normal wurden. "Höflich' benahm man sich, wenn man sich ,wie bei Hofe' benahm. Nun sind die Höfe schon längst kein Ort einer besonderen Höflichkeit mehr, das Wort ist aber noch da und kann als nunmehr direkte Bezeichnung für ein respektvolles Benehmen gebraucht werden (hier gleich wieder: Respekt heißt Rücksicht, wird bei Rücksicht zurückgesehen?).

Wer sich aufmacht, in einem Text Metaphern zu identifizieren, der hat ständig eine Entscheidung zu treffen zwischen verbrauchter und noch aktiver (d. h. eine Bedeutungsübertragung bewerkstelligender) Metapher. Dabei steht sehr wenig fest, es gibt fast beliebig große Spielräume, so dass sich die Entscheidungen kaum je als Behauptungen begründen lassen. Derartige Behauptungen müssten aber begründbar sein, mehr noch, sich eigentlich von selbst verstehen, wenn die gegebene Charakterisierung der Metapher zur empirischen Erhebung von Metaphern-Vorkommnissen in Texten tauglich sein soll. Werfen wir einen Blick auf das Procedere:

In der Analyse des Beispieltextes "Königin Beatrix. Opfert sie ihr Herz für die Krone?" (95/83) wird im Satz ,Prinz Claus von Amberg, Hollands amtierender Prinzgemahl, ist zurückgefallen in seine Schwermut' der Ausdruck ,zurückfallen' als Metapher gezählt. Tja, man „fällt” anscheinend im "eigentlichen Sinne" nicht in eine Krankheit zurück, schließlich ist man selbst doch nicht höher oder weiter als sie? Aber ist das etwa eine sinnvolle Überlegung? Hören wir im Gebrauch des Wortes ,Rückfall' im Zusammenhang mit einer Krankheit, dass da irgendetwas "fällt"? Und weshalb ist denn dann "Schwermut' keine Metapher? Hat ein Mut, hat das Gemüt etwa ein Gewicht? Einige Sätze später heißt es "Die Depression ist bleibend, nur durch Medikamente zu unterdrücken." Dabei wird ,unterdrücken' als Metapher gezählt. Man darf sich also fragen, wie man "normal” ausdrücken möchte, dass eine Krankheit durch ein Medikament, ja was denn, ,ertragbar'? - ist das keine Metapher? - wird. Ein Satz später wird über diese Depression gesagt, sie "trifft einen Mann, der nie ein Temperamentsbolzen war ..." und dabei ist dann ,Temperamentsbolzen' eine Metapher und ,trifft' ist keine. Wie kann eine Depression einen Mann "treffen"? Wie sich Personen auf der Straße treffen? Wie ein Schütze die Scheibe trifft? Nein, sollen wir hier akzeptieren: Ganz direkt, so wie eben eine Depression einen Mann trifft. Kurz: Die Entscheidungen sind alle irgendwie möglich, aber eindeutig und zwingend ist kaum eine.

Soviel vorerst zur Identifikation der Metaphorik in der RegenbogenPresse. Kann es sein, dass dort eben lockerer gesprochen wird, also eine 
Analyse auch großzügig sein darf - zumal deren Auswertung auch nicht die Brisanz einer rechtstheoretischen Ideologiekritik bekommt?

Schauen wir also zu den Rechtstextanalysen in der 96er-Studie. Hier scheint Sobota am Ziel zu sein. Die Befunde sehen solide aus und sie scheinen die These, dass an wichtigen Stellen der argumentative Gehalt der Texte ab- und der pathetische zunimmt, eindringlich zu belegen. Ich überprüfe zwei Stellen aus höchstrichterlichen Urteilen, an denen die entsprechenden Verhältnisse klar zu sein scheinen.

Die erste Stelle ist aus dem berühmten BVerfG-Urteil "Solange II" vom 22. Oktober 1986. Sobota gibt mehrere Diagramme für das LogosEthos-Pathos-Geschehen (96/125, Abb. 2,3; 96/126, Abb. 4) und schreibt kommentierend zu einer besonders brisanten Passage, nämlich dem Abschnitt B.II.1.a) des Urteils, Folgendes:

„Sobald es (= das Gericht, H. W.) ... zu den Grundrechten der Beschwerdeführer kommt, eskaliert das Pathos bei rapide abnehmender Argumentation. Der vorläufige Höhepunkt wird mit dem Verweis auf Art. 24 Abs. 1 GG erreicht ... von dem es heißt, er ermögliche, die deutsche Rechtsordnung, derart zu öffnen (Metapher)', dass , Geltung und Anwendbarkeit eines Rechts aus anderer Quelle (Metapher) innerhalb dieses Hoheitsbereichs Raum gelassen wird (Metapher)'." (96/127)

Was wird hier mitgeteilt? Sobota diagnostiziert, es sei kaum Argumentation da, dafür eskaliere das Pathos. Nehmen wir die Behauptung über das eskalierende Pathos zuerst. Sie ist damit begründet, dass hier drei Metaphern fast unmittelbar aufeinander folgen. Bei der Identifikation von Metaphern tritt das oben erörterte Entscheidungsproblem auf. Schauen wir $\mathrm{zu}$, welche Entscheidungen $\mathrm{zu}$ akzeptieren sind, damit Sobota Recht hat. Wieso ist das "Öffnen" einer Rechtsordnung eine Metapher? Lässt sich eine Rechtsordnung nicht „öffnen"? Etwa deshalb nicht, weil sie keine Türe hat? Was lässt sie sich denn? Erweitern? Zugänglich machen? Verflixt, ist das nicht auch alles räumlich, insofern metaphorisch? Nehmen wir die nächste Metapher: da wird von einem Recht aus anderer "Quelle" gesprochen. Sehen wir es nicht geradezu sprudeln? Aber nein, niemand weiß mehr, wie eine Quelle aussieht, und jeder versteht direkt, dass "Quelle" hier dasjenige bezeichnet, woher etwas kommt. Ebenso die dritte Metapher: „Raum lassen”. Da wird über die Rechtsordnung wieder wie über etwas Räumliches geredet, obwohl sie doch wahrlich nicht räumlich ist.

Aber was für ein "Gegenstand" ist sie denn „eigentlich"? Dies dürfte die Crux bei der Sache sein: Eine Rechtsordnung ist überhaupt kein Gegenstand, der im Raum angetroffen werden kann, sie ist etwas Abstraktes. Und was für ein Vokabular haben wir, um abstrakte Gegenstände zur Sprache zu bringen, ohne dabei „übertragend" zu reden? Oder kommen am Ende Abstrakta in der Normalsprache gar nicht vor? Aber 
gewiss doch: Es ist ganz normal, dass über abstrakte Gegenstände, z. B. über Pläne, Ziele, Gedanken, Werte usw. gesprochen wird. Und weil die eben nicht konkret sind, wir aber unter Umständen über sie etwas Konkretes sagen wollen, deshalb benutzen wir Prädikate, die ursprünglich einmal übertragend waren, die aber, weil sich andere Möglichkeiten kaum anbieten, ohne weiteres gewöhnlich und direkt verstanden werden. So sind Ziele aussichtsreich (wie Berggipfel?), Gedanken sind klar (wie Wasser?), Werte sind schutzbedürftig (wie Kinder?) und Pflichten sind hart (wie Steine).

Fazit: Die Aussagen, die das Gericht an der von Sobota analysierten Stelle macht, sind ganz normale Prädikationen über den abstrakten Gegenstand "Deutsche Rechtsordnung", in die eine metaphorische Bedeutung nur nachträglich und mit einer gehörigen Portion Eigensinn hinein projizierbar ist. Von dieser Art ist hier die "Pathos-Eskalation".

Gleichzeitig und konterkarierend soll die Argumentation „rapide abnehmen". Wenn ich für diese Aussage den oben diskutierten Argumentbegriff unterstelle, wären also an der zitierten Stelle wenige oder gar keine „X weil X"-Konstruktionen, Kodifikationsprämissen, Rechtsgrundsatzprämissen, Alltagsprämissen oder Folgerungen zu finden. Das ist auch tatsächlich der Fall, es besagt aber leider nichts darüber, wie hier argumentiert wird. Um die Verhältnisse im Text für den Leser möglichst übersichtlich und einschätzbar zu machen, beschreibe ich hier das Wichtigste aus der fraglichen Passage. Wer es nachprüfen möchte, findet den Abschnitt im Anhang abgedruckt.

Zunächst einmal: In demselben Satz, der die drei „Metaphern” enthält, gibt es einen Hinweis auf Art. 24 Abs. 1 GG und nicht weniger als drei Hinweise auf BVerfG-Entscheide. Nach Sobotas Charakterisierungen hätten alle vier Hinweise (einmal Logos, dreimal Ethos) Argumentcharakter. Also in einem Satz, auf fünf Zeilen, wären das vier Argumente gegen die drei Figuren. Ist das "rapide abnehmende Argumentation"? Gleich der nächste Satz enthält wieder einen Hinweis auf 24 Abs. 1 GG, dann folgen 13 Zeilen, in denen bei Befolgung von Sobotas Anweisungen kein Argument zu finden ist. In den letzten 11 Zeilen des Absatzes gibt es dann wieder drei Hinweise auf GG-Artikel, einen Hinweis auf einen Artikel des EWG-Vertrags und am Schluss eine "Folgerung” („Aus dem Rechtsanwendungsbefehl ... ergibt sich ...). Bei Zugrundelegung von Sobotas Argumentbegriff ergeben sich damit insgesamt 10 Argument-Vorkommnisse. Der Abschnitt ist 29 Zeilen, also ca. 290 Wörter lang, es errechnet sich eine Argument-,Dichte" von 34,5 auf 1.000. In Sobotas Darstellung (96/126, Abb. 4) sieht das nun ganz anders aus. Da liegt der Argumentanteil zunächst bei über 20 und fällt dann auf unter 10. Teilweise erklären sich diese Unterschiede wohl 
daraus, dass sie als Bezugseinheiten die Halbseiten der Originalschrift genommen hat (der von mir benutzte Text ist anders paginiert). Die Halbseite von Solange II hat (in meiner Paginierung) ca. 250 Wörter, und möglicherweise lag der Abschnitt B.II.1.a) bei Sobota in zwei Halbseiten. Zur Gänze erklären sich so erhebliche Differenzen daraus nicht, aber darüber will ich hier nicht weiter rechten. Mein Punkt ist, dass für das Durchschauen der Argumentation der Bezug auf Halbseiten ganz und gar unzweckmäßig ist. Er ist bestens geeignet, das argumentative Geschehen im Text zu verfehlen, da sich dieses nun einmal nicht an irgendeinen zufälligen Satzspiegel hält. Der zitierte Abschnitt B.II.1.a) ist dafür ein deutliches Beispiel. Er enthält eine luzide Argumentation, die sich aber nur erschließt, wenn man den Absatz in seiner Gesamtheit betrachtet und analysiert. Für die Einlösung dieser Behauptung ist es nicht nötig, eine regelrechte, differenzierte Argumentationsanalyse vorzulegen. Es reicht, nur grob die Abfolge der Argumentationsschritte anzugeben. Es sind sieben:

1. Art. 24 Abs. 1 GG ermöglicht die Öffnung des Rechts der BRD.

2. Diese Möglichkeit ist in drei BVerfG-Entscheiden bestätigt und bekräftigt worden.

3. Um diese Möglichkeit zu verwirklichen, bedarf es eines „innerstaatlichen Rechtsanwendungsbefehls".

4. Ein solcher kann auch durch Verträge, die "Hoheitsrechte auf zwischenstaatliche Einrichtungen übertragen", erfolgen.

5. Dieses ist durch die Zustimmung der BRD zum EWGV geschehen.

6. Die Zustimmung ist verfassungskonform gegeben worden.

7. Daraus ergibt sich die "unmittelbare Geltung der Gemeinschaftsverordnungen für die BRD und ihr Anwendungsvorrang gegenüber innerstaatlichem Recht". (Alle Zitate aus: Solange II, Abschnitt B.II.1.a.)

Ich stelle noch einmal fest: Diese Argumentation enthält keine (explizite) "X weil $X$ " - Konstruktion, keine (expliziten) Kodifikations-, Rechtsgrundsatz- oder Alltagsprämissen, aber sie enthält eine Sequenz von Argumentationsschritten, die sich dem verständigen Leser ohne weiteres erschließt. Dass diese Sequenz als Argumentation ausreichend ist, dass sie nicht schwach, nicht "rapide abnehmend" ist, sondern dass sie die anvisierte Konklusion rational erreicht, ist offenbar.

Sobota stellt die Sache so dar, als würde das Resultat gerade nicht durch Argumentation, sondern durch Pathos erreicht. Den Beweis dafür sollen wir zunächst einmal in der besagten Metaphernhäufung se- 
hen. Ich habe oben gesagt, was das für "Metaphern" sind. Aber können etwa diese drei "Metaphern", wenngleich in einem Satz, für die Diagnose "eskalierendes Pathos" ausreichen? Im Diagramm (96/126, Abb. 4) haben wir einen Pathos-Gipfel von über 80 . Das bedeutet, es müssten in der Umgebung dieser drei "Metaphern" ca. weitere 20 Figuren zu finden sein! Nur wo? Wenn ich als Normalgebildeter die Passage lese, finde ich überhaupt keine rhetorische Figur. Nehme ich Sobotas Figurenbeschreibungen dazu, dann könnte ich außer den drei besprochenen noch ca. sieben weitere Figuren finden (dazu muss ich dann das "Beimessen" und "Beilegen" eines Vorrangs als zwei Metaphern lesen, ebenso das "Übertragen" von Rechten, das "Sich-Erstrecken" eines Befehls auf einen Sachverhalt und das "Inkorporieren" von Recht; sodann müsste ich evtl. das eingeschobene "auch der hier in Rede stehenden

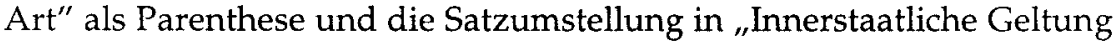
usw." als „Figuren" in Sobotas Sinne zählen). Damit käme ich dann auf ca. zehn Figuren, die in der Passage zu finden wären. Es stünde gleichsam 10:10. Sollten diese Figuren wirklich einen Gefühlsappell ausdrücken, wäre der genau gleichstark wie der Verstandesappell. Was für eine Ausgewogenheit - Leib und Geist wären in perfekter Balance! Keine Rede wäre von einer Ersetzung des mangelnden Logos durch Pathos. Und dieses Resultat ergäbe sich, wohlgemerkt, bei weitgehender Übernahme der Vorgaben von Sobota. ${ }^{11}$ Ohne diese Vorgaben finden wir in dem fraglichen Abschnitt B.II.1.a) eine sinnvolle Argumentation für eine Subthese innerhalb des Solange II-Urteils, die in einer eher nüchternen, von zahlreichen Hinweisen auf juristische Normen begleiteten Sprache daherkommt. Sie entspricht völlig den üblichen Erwartungen an juristische Texte: trocken, rational, argumentativ.

Die zweite Stelle, die ich im Hinblick auf Sobotas These näher betrachten möchte, stammt aus BVerfGE 65, 9. Hier hat am 11. Oktober 1983 der Erste Senat seine ordnungsgemäße Besetzung behauptet und diese Behauptung auf knapp acht Seiten begründet. In Sobotas 96erStudie wird dazu ein Logos-Ethos-Pathos-Diagramm gezeigt (96/123, Abb. 1). Im Kommentar zum Diagramm wird erneut die Bestätigung der These festgestellt. Sobota schreibt, dass hier "trotz der prozessualen Thematik, die große Nüchternheit verspricht, ... das Gericht an vielen Stellen häufiger rhetorische Figuren als Argumente einsetzt". (96/124) Allerdings seien das bevorzugt "Stilmittel, die einem Pathos der Sachlichkeit (z. B. Doppelpunkte, Einschübe, betonende Satzumstellungen, emphatischer Satzneubeginn statt Komma)" entsprächen. (ebd.) Als Le-

${ }^{11}$ Genau genommen fällt mein Resultat noch zu Sobotas Gunsten aus, weil meine Berechnungen sich nicht auf eine Halbseite, sondern auf die gesamte (in meiner Paginierung 29 Zeilen lange) Passage bezogen haben. 
ser stolpere ich natürlich jetzt wieder über den eigenwilligen Sprachgebrauch: "Statt" Nüchternheit soll es hier rhetorische Figuren, aber keine emotionalen, sondern sachliche geben.

Immerhin käme aber dieses sachliche Pathos mit dem von ihr als typisch befundenen rhetorischen Schwung daher, insbesondere mit einem Doppelgipfel in der Endphase der Pathos-Kurve. Ich nehme jetzt den ersten Höhepunkt dieses Doppelgipfels, er liegt über der Halbseite 156/1, unter die Lupe. Dort soll wieder die Logos- und die PathosDichte besonders weit auseinander klaffen. Inhaltlich befindet sich der Senat bei der Begründung einer Subthese: Der Bundesrat habe einen Bundesrichter in den Ersten Senat zu wählen gehabt.

Sobotas Diagramm weist für die Stelle eine Pathos-Dichte von über 60 aus, während Logos (bzw. Ethos) unter 10 steht. Es müssten sich also (hier hat die Halbseite 140 Wörter) acht Figuren und ein Argument finden lassen. Nach Ansicht der Autorin enthält die Passage eine „entscheidende Begründung", diese sei aber zu wenig gestützt, weil hier "nur ... ein Textargument" (96/124) aufgeboten wird. Zur Erinnerung: Ein "Textargument" ist ein Ethos-Argument. Ob ein Ethos-Argument schwächer ist als ein Logos-Argument, das ist in Wahrheit eine inhaltliche Frage; desgleichen ob "ein" Argument schwächer ist als mehrere. An dieser Stelle soll die Schwäche des Arguments darin liegen, dass es kein Gesetz für die Bestimmung gebe, die Aufteilung der Wahlberechtigung von zwei bzw. einem Bundesrichter auf die beiden dafür zuständigen Wahlorgane Bundestag und Bundesrat, solle einfach der weiteren Entwicklung vorbehalten bleiben, sondern nur einen Beschluss des damals befassten Bundestags-Ausschusses. Und diese argumentative Schwäche müsse eben durch stilistischen Nachdruck kompensiert werden.

Was ist davon zu halten? Um die Sache auch hier für den weniger beteiligten Leser nicht unnötig lang zu machen, bringe ich wieder nur eine Beschreibung der Passage. Wer die Sache überprüfen will, findet die Stelle im Anhang abgedruckt.

Zunächst zum argumentativen Gehalt: Die Argumentation für die fragliche Subthese beginnt genau eine Halbseite früher, nämlich am Kopf von Seite 155/2, und reicht über die inkriminierte Passage hinaus bis in die Mitte von Seite 156/2. In der fraglichen Halbseite 156/1 ist, für sich betrachtet, überhaupt keine Argumentation auszumachen. Dort wird berichtet, dass das Gesetz etwas nicht festgelegt hat, was, wie sich der Ausschuss geeinigt hatte, die faktische Entwicklung festlegen sollte. Sodann wird berichtet, wie die faktische Entwicklung verlief. Die argumentative Funktion dieser Berichte für die Begründung der Subthese wird wie gesagt bloß deutlich, wenn die gesamte Argumentation 
zu dieser Subthese von Anfang 155/2 bis Mitte 156/2 betrachtet und analysiert wird. Es zeigen sich dann sechs Schritte:

1. GG und BVerfGG 1951 hatten festgelegt, dass pro Senat zwölf Richter, davon vier Bundesrichter, von Bundesrat und Bundestag paritätisch zu wählen seien.

2. Das Änderungsgesetz 1956 legte dann fest, dass Bundestag und Bundesrat im Verhältnis 2:1 drei Bundesrichter wählen. Wer aber zwei und wer einen wählt, dazu sagte es nichts.

3. Man hat sich darüber im zuständigen BT-Ausschuss geeinigt: Das Verhältnis solle sich in der tatsächlichen Entwicklung von selbst herausbilden.

4. Die tatsächliche Entwicklung hat es so eingerichtet, dass der Bundesrat das Wahlorgan ist, welchen einen Bundesrichter wählt.

5. An dieser Rechtslage hat sich durch das Änderungsgesetz 1970 nichts geändert.

6. Also wählt der Bundesrat einen Bundesrichter.

Innerhalb der in Frage stehenden Halbseite 156/1 liegen die Schritte (2) und (3) und ein Teil von Schritt (4). Es gibt zwei Hinweise auf Gesetze und einen auf den Bericht des BT-Rechtsausschusses. Die Frage, weshalb Sobota hier nicht drei, sondern nur ein Argument findet, kann ich nicht beantworten. Zur Frage, weshalb sie ihr einziges gefundenes Argument, nämlich den Hinweis auf den Ausschussbericht, als schwach diagnostiziert, verweist sie auf ein "Positivierungsdefizit”. Offenbar soll also ein Beschluss eines BT-Ausschusses ein schwächeres Argument sein als ein Gesetz. Aber ob das so ist oder nicht, das ist, wie schon gesagt, eine Frage, die vollständig von den Inhalten abhängt, um die es dabei geht. Wenn Sobota der Ansicht ist, dass für eine so entscheidende Festlegung ein BT-Rechtsausschuss nicht genügend kompetent ist, dann wäre das ein inhaltlicher Einwand, den sie gegen diese Stelle in der Urteilsbegründung vorbringen würde. Damit der Einwand die Begründung wirklich schwächen oder aushebeln könnte, dazu müsste er inhaltlich richtig sein. Und dieses müsste gezeigt werden. So sieht sie also aus, die Sachlage, bei der Sobota diagnostiziert, es gebe hier ein an formalen Kriterien ("nur ein Textargument") erkennbares argumentatives Defizit.

Werfen wir noch einen Blick auf die Pathos-Analyse. Es müssten acht Figuren zu finden sein. Wenn ich "Wahlorgan” als Metapher nehme (und, weil das Wort zweimal vorkommt, als zwei Figuren zähle?), sodann die eingeschobene Klammer und den Doppelpunkt be- 
rücksichtige, käme ich auf vier Figuren. Mit einem Gefühlsappell, um die vorgeblich mangelnde Rationalität $\mathrm{zu}$ kompensieren, haben alle vier kaum etwas zu tun. Und wo sollen die übrigen vier Figuren versteckt sein?

Auch mit Sobotas Vorgaben hätte ich also einen argumentativen Gehalt, der dem Pathos-Gehalt durchaus das Wasser reichen könnte, insbesondere, weil es sich eher um "sachliches Pathos" handelt. Zu dieser speziellen Pathos-Sorte möchte ich bei dieser Gelegenheit noch einmal eine kleine Bemerkung machen: Genau wie schon in der Solange IIPassage ist auch die hier betrachtete Stelle in einem Stil gehalten, den jedes normale Lesen als einen ganz gewöhnlichen juristischen Text identifizieren würde. Es gibt zahlreiche Referenzen auf juristische Normen, es gibt nüchterne, sachliche Aussagen, viele papierene Redewendungen. In der 92er-Studie hatte Sobota eingeräumt, "dass in der Rechtssprache manches als normal erscheint, was in der Umgangssprache schon als abweichend gewertet würde" und dass es insofern bei der Figurenerfassung noch „einige Zweifelsfragen” gebe (92/233). Sollen wir dem etwa entnehmen, dass dem normalgebildeten und normalsprachlichen Leser ein Urteil hinsichtlich der Figurenerfassung in Rechtstexten gar nicht zugestanden werden kann, weil er z. B. keine Ahnung hat, welche Wortfolgen in den Ohren von Juristen normale und welche sachlich-pathetisch umgestellte Sätze sind?

Vielleicht sind ja die Juristen wirklich eine Menschengruppe mit ganz eigenen Sprachgewohnheiten. Sobota möchte glauben machen, das BVerfG habe in seinen Urteilen - über ganz verschiedene Angelegenheiten, zu ganz verschiedenen Zeiten und teilweise von ganz verschiedenen Richtern verfasst - eine "Handschrift", d. h. das Gericht würde in einer bestimmten immergleichen Form rhetorische Figuren über den Urteilstext verteilen. Schon in der 92er-Studie hieß es, die Pathos-Kurven wiesen "alle ein gemeinsames Muster" auf (92/234). Und in der 96er-Studie haben sich die Ansichten über dieses gemeinsame Muster so verfestigt, dass vom "typischen Doppelgipfel" (nebst amerikanischer Benamung als "Twin Peak") der "Sobota-Spule" gesprochen werden kann. Wenn der Leser sich die Kurven anschaut ohne sich von diesen Vorschriften an die Hand nehmen zu lassen, fällt es ihm aber nicht leicht, das zu sehen. Sicher, es ist immer eine Zackenkurve da, die die Pathos-Höhe darstellen soll. Aber um von einem gemeinsamen Muster sprechen zu können, dazu muss man seine Abstraktionskraft schon gehörig anstrengen. Sollte das nicht möglich sein? St. Exupery hat in seiner Erzählung vom „Kleinen Prinzen“ an einem amüsanten Beispiel gezeigt, dass bei gehörigem Einsatz von Abstrakti- 
onskraft eine so aufregende Sache wie eine Schlange, die einen Elefanten verschlungen hat, einfach einem alten Schlapphut gleicht.

Die Kurven sind, vorsichtig gesagt, doch recht verschieden und auch der typische Doppelgipfel ist nicht immer vorhanden. Das ist der Autorin auch nicht etwa einfach entgangen. Denn wenn die Kurve zu stark vom "gemeinsamen Muster" abweicht, dann sucht sie dafür Erklärungen: In BVerfGE 82, $126 \mathrm{zu}$ "Kündigungsfristen" ist die Kurve deutlich anders (96/130, Abb.7), also der Pathos-Einsatz ist nicht wie angeblich sonst immer verteilt, und zwar weil - man muss ordentlich die Augen aufreißen vor Staunen - "das Gericht von seiner eigenen Arbeit gelangweilt war" (96/131). Tja, wer sich langweilt, der hat keinen Spaß mehr am Einsatz von Stilmitteln, weder von bezaubernden noch von sachlich-distanzierenden? In der Wissenschaftstheorie heißt so etwas eine "Ad hoc-Erklärung": Eine falsifizierende Instanz wird wegerklärt, indem man eine neue Hypothese einführt (hier: Wer sich langweilt, macht keinen ordentlichen Gebrauch von Pathos), die jedoch den Anteil an zu prüfender Theorie nicht senkt, sondern vermehrt. Wer zu solchen Mitteln greift, kann allerdings beim Vormarsch mit seiner Hypothese nicht aufgehalten werden, riskiert aber, dass sein ganzes Forschungsprogramm "degeneriert".12

Ich komme zum Fazit dieser Überprüfung des Analyseverfahrens: Bei einer Analyse, die sich nicht auf Sobotas Ziel verpflichtet hat, an wichtigen Stellen möglichst viel Pathos bei möglichst wenig Logos zu sehen, lassen sich ihre Befunde auch nicht annähernd reproduzieren. Es kommt eher das genaue Gegenteil heraus: In den höchstrichterlichen Urteilen ist bei kaum nennenswertem rhetorischen Appell ein durchaus hinreichender argumentativer Gehalt anzutreffen (damit ist allerdings noch nichts darüber gesagt, wieweit die Argumentationen die Urteile, welche sie begründen, auch als gültig ausweisen).

\subsubsection{Beurteilung des Analyseverfahrens}

Die Überprüfung der von Sobota erhobenen Daten ist hier nur stichprobenartig vorgeführt werden. Dieses Material reicht aber aus, um gravierende methodische Zweifel am Verfahren zu äußern. Das „Messgerät", also die Beschreibungen, Kriterien und Illustrationen, mit denen

\footnotetext{
12 Der Wissenschaftstheoretiker Imre Lakatos hat das an der schönen Illustration mit dem vor-Einstein'schen Physiker gezeigt, der zur Erklärung einer Gegenevidenz gegen die Newton'sche Mechanik einen Planeten postuliert, dann, als der sich nicht finden lässt, eine kosmische Wolke, die ihn verbirgt usw.; und der niemals zum Aufgeben seiner Hypothese gezwungen werden kann. Vgl. IMRE LAKATOS, Falsifikation und die Methodologie wissenschaftlicher Forschungsprogramme, in: IMRE LAKATOS/ALAN MUSGRAVE (Hrsg.), Kritik und Erkenntnisfortschritt. Braunschweig 1974, 89-189, dort 98 f.
} 
Textvorkommnisse als Exemplare von „rhetorische Figur" bzw. „Ethos-" oder "Logos-Argument" identifizierbar sein sollen, ist so ungenau, dass sein Einsatz jeweils einen großen Spielraum für Entscheidungen lässt, Entscheidungen, die von Forscher zu Forscher unterschiedlich getroffen werden können. Das kann große Verschiedenheiten bei den erhobenen Daten zur Folge haben, Verschiedenheiten, die es erlauben, in der Auswertung zu anderen, im Extremfalle sogar zu gegenteiligen Einschätzungen zu gelangen. Dieses bedeutet aber: Das Verfahren erfüllt nicht die Objektivitätsforderung, die für wissenschaftliche Messgeräte erhoben wird.

Was hat das für Konsequenzen? Wie schon erwähnt, ist die Objektivitätsforderung die grundlegende. Wenn sie nicht erfüllt ist, sind auch die anderen beiden nicht erfüllt. Ein nicht-objektives Verfahren ist nicht reliabel, ein nicht reliables nicht valide. Nun gibt aber Sobota an, dass zumindest die Pathos-Daten auf Reliabilität und Validität überprüft seien (95/81, 96/122, Anm. 44 und 45).

Wie kann das sein? Meine Erklärungsversuche sind vielleicht nicht sonderlich originell, aber weil mir Interna aus Sobotas Forschungen nicht bekannt sind, muss ich mit nahe liegenden Überlegungen arbeiten: Wenn jemand beim Einsatz eines textwissenschaftlichen Analyseapparats sein Textverständnis und seine Intuitionen aktivieren muss und dabei einen mehr oder weniger großen Spielraum für die Identifikation dessen hat, was er findet, dann dürfen wir annehmen, dass er diesen Spielraum loyal nutzt. Nehmen wir weiter an, dass er die Hypothese kennt, die hier getestet werden soll. Er weiß, dass es um die Widerlegung der rationalen Rechtstheorie geht und dass ganz allgemein Pathos wichtiger sein soll als Logos. Ohne jetzt auch nur die geringsten Täuschungsabsichten zu unterstellen, dürfen wir die folgende Vermutung bilden: Die durch das Verfahren vorgegebenen Spielräume werden dazu führen, dass die "Rater" soweit wie irgend möglich die Textwirklichkeit in etwa so vorfinden werden, wie es die Hypothese besagt.

Diese Überlegung ist schlicht, aber nicht unsinnig, und sie reicht aus, um zu erklären, wie Sobotas Pathos-Messverfahren als reliabel erscheinen kann. Verschiedene "Rater” kommen beim Einsatz des Messgeräts zu ähnlichen "Daten", weil sie sich im Hinblick auf die Kenntnis der Hypothese und die Loyalität mit ihr ähneln. Auf diese Weise können ihre Resultate reproduzierbar werden, doch das ist ein Schein, denn jemand, der nicht loyal mit der Hypothese ist, kann, wie ich oben gezeigt habe, zu ganz anderen Resultaten gelangen.

Es ist aber auch noch zu bedenken, dass diese Reliabilität nicht für das ganze Verfahren, sondern nur für den Pathos-Teil überprüft worden ist. Die Identifikationsvorschriften für Argumente enthalten, wie 
oben referiert, so große Ermessensspielräume, dass sie genau genommen gar keine Datenerhebung anleiten können, sondern, wie die Autorin selbst sagt, allenfalls eine "Rekonstruktion" dessen, was gemeint sein könnte, wobei selbst die noch auf "Intuition", „Phantasie“ und den "gutwilligen Leser" angewiesen ist (96/120). Ich vermute daher, dass hier eine Reliabilitätsprüfung, auch wenn die gleichen subjektiven Bedingungen bei den Ratern vorliegen wie bei der Pathoserhebung, bedeutend niedrigere Werte erbringen würde. Wie steht es mit der dritten Forderung, der Validität?

Für den Validitätstest wird geprüft, ob das Verfahren das erfasst, was es erfassen sollte. Weil das, was es erfassen „sollte”, mit den begrifflichen Mitteln des Vorverständnisses artikuliert worden ist, besteht die Prüfung im Wesentlichen in einem Vergleich der noch nicht operationalisierten Beschreibung mit der durch das Messverfahren ermöglichten Beschreibung. Sobota gibt an, die Validität, und zwar wiederum die der Pathos-Erhebungen, geprüft zu haben. Sie habe die gemessene Pathos-Dichte von Texten mit der empfundenen Emotionalität vergleichen lassen; und dieser Vergleich habe eine hohe Übereinstimmung erbracht. Es wurden dazu zwei Lesern Texte vorgelegt mit der Aufforderung, deren Emotionalität in einer Skala mit vier Rangstufen (nüchtern, moderat, bewegt, hoch affektiv) einzuordnen. Und es zeigte sich: Beide ordnen in hoher Übereinstimmung in etwa so ein, wie gemessen wurde. (96/122, Anm. 45) Was ist dazu zu sagen?

Wenn ich das so übernehmen müsste, würde es mich verblüffen, ich würde zu überlegen beginnen, ob das Zufall sein soll oder was es bedeuten könnte. Vielleicht muss ich das aber gar nicht übernehmen, wenn ich sehe, dass Sobota selber über die Bewertungen ihrer PathosMaßzahlen durchaus unterschiedliche und kaum miteinander verträgliche Aussagen macht.

In der 95er-Studie berichtet sie, es hätten sich folgende Bewertungen ergeben: Eine Pathos-Dichte von 40 würde als "nüchtern" empfunden, eine von 70 als "noch sachlich, aber schon lebendig", eine von 90 als "nicht mehr sachlich, sondern gefühlvoll" und eine von 120 als "hochaffektiv" (95/81). Einige Seiten nach dieser Information werden die Verhältnisse genauso in einem Diagramm abgebildet (95/84, Abb.13). Wieder einige Seiten später findet sie es nicht verwunderlich, dass Presseartikel über die Herz-Kreislauf-Thematik „im Hinblick auf die hohe Affektivität des Stoffes Herz" (95/86, Hervorhebung im Text) in "gefühlsstarker Weise berichten" (ebd.). Diese Artikel wiesen "den vergleichsweise hohen Figurenanteil von 36 auf" (ebd.). Was ist jetzt los? Bis 40 sollte es doch "nüchtern" sein? Ist das ein Druckfehler? Aber über den Stil des BVerfG hatte sie auch geschrieben, der weise "eine so 
hohe rhetorische Intensität ... (auf), dass Prädikate wie ,trocken' und ,nüchtern' unangebracht wirken. Der Figurenanteil liegt etwa bei $46,5^{\prime \prime}$ (92/233). Also bitte: 46,5 , das wäre knapp über „nüchtern“, noch sehr weit entfernt von 70 - „noch sachlich, aber schon lebendig" und geradezu meilenweit entfernt von 90 - „nicht mehr sachlich, sondern gefühlvoll". Wie ist das zu erklären? Ich denke folgendermaßen: Die "Figurendichte" ist ein Quotient aus Figurenanzahl : Textlänge (gemessen als Wortanzahl des Textes), welcher auf 1.000 Wörter bezogen wird. Dieser Quotient ergibt natürlich nur dann vergleichbare Werte, wenn die Textlänge gleich ist. Das ist aber (obwohl Sobota sich dieser Problematik bewusst ist, vgl. 92/234, Anm. 19) leider nicht der Fall. Bei den analysierten Presseartikeln ist die (je verschiedene) Länge das Textlängenmaß, bei juristischen Texten ist es die „Halbseite”, die 137 bis 150 Wörter habe (das allein ergibt Schwankungen von $10 \%$ !). Wenn sie aber irgendwo besonders viele Figuren entdeckt, dann gefällt es ihr unter Umständen, einen besonders eindrucksvollen Pathos-Wert zu liefern, indem sie eine beliebige Passage aus dem Text herausschneidet. So ermittelt sie einen Pathos-Wert von 110, indem sie einen Satz mit 18 Wörtern auszählt $(95 / 89)$, oder gar von 357 (!), indem sie aus einem laufenden Text eine Passage von 14 Wörtern, die fünf Figuren enthalten soll, ausschneidet (95/96). Hier bekomme ich dann langsam Zweifel, ob das überhaupt ernst gemeint ist. Es ist doch eine Trivialität, dass sich bei einem Text, in dem man die Figurenanzahl ausgezählt hat, allein dadurch, dass man figurenlose Teile weglässt, die so verstandene „Figuren-Dichte" beliebig erhöhen lässt. Wenn sie von den genannten 14 Wörtern, in denen sie fünf Figuren ermittelt hat, eine Dichte von 357 errechnet, dann könnte sie im letzten Halbsatz dieser 14-Wort-Passage, die nämlich vier Wörter lang ist und zwei von ihren Figuren enthält, also eine Dichte von 500 ermitteln. (Wie das wohl bewertet würde, wenn 120 schon als "hochaffektiv" empfunden wird?)

Mit diesen Hinweisen und Überlegungen hoffe ich hinreichend begründet zu haben, weshalb ich dem erfolgreichen Validitätstest keine sehr große Beweiskraft zuzubilligen bereit bin.

Und damit komme ich zur Beantwortung der in dieser wissenschaftstheoretischen Kritik leitenden Frage, ob Sobotas Rechtstextanalysen empirisch sind. Aus dem Gesagten ergibt sich eine negative Einschätzung. Das Verfahren hat gewisse Ähnlichkeiten mit empirischer Forschung, erreicht aber nicht die Standards und Forderungen, die für die intersubjektive Überprüfbarkeit der Ergebnisse einzuhalten wären.

Mir scheint, Sobota hat geahnt, dass ihre Untersuchungen bei hinreichender Aufmerksamkeit seitens ihrer Leser unter einen solchen Verdacht geraten können. Denn sie bringt mehrere abgeklärt wirkende 
Aufforderungen, die Leser mögen die Erwartungen an ihre Forschung zurückschrauben. So heißt es: „,... empirische Untersuchungen (können) keine Wahrheiten zutage bringen" (96/119), sondern nur "einige Bezugspunkte definieren, um dann deren Relationen ... zu beschreiben" (ebd.). Das, findet sie, sei „eine sinnvolle Einsicht: Die Beziehungen, die zwischen den Kategorien festgestellt wurden, gelten" (ebd.). Was soll daran sinnvoll sein? Wenn die "Kategorien" so vage und offen bestimmt werden, wie hier bei Sobota die "rhetorische Figur" und das "Argument", dann können in hohem Maße genau solche "Beziehungen" zwischen ihnen "festgestellt" werden, wie man sie sich gewünscht bzw. in seiner Hypothese formuliert hatte. Wenn jemand "Messungen" durchführt mit Maßstäben, die nicht starr sind, also z. B. nicht aus Holz oder Metall bestehen, sondern aus einem elastischen Material, aus Gummi, Teig, Schlingpflanzen (mir fällt das Bild ein, das Lewis Caroll in "Alice im Wunderland" zeichnet, wo ein Krocketspiel stattfinden soll, mit Flamingos als Schlägern, deren Hals sich selbst bewegt, und Igeln als Bällen, die sich selbst fortbewegen), dann sind für mögliche "Messresultate" zwar auch noch gewisse Grenzen angebbar. Doch niemand würde diese Resultate schon deshalb "sinnvoll" nennen.

Eine Erhebung, die Gutwilligkeit, Intuition und Phantasie erfordert, muss nicht grundsätzlich unwissenschaftlich sein, ist aber jedenfalls keine Empirie. Dass dann die erzeugten Ergebnisse in eindrucksvollen Schaubildern dargestellt und über diese Schaubilder weitere Theoreme (Sobota Spule, Twin-Peak-Formation etc.) formuliert werden, das trägt diesen Ergebnissen eine sekundäre, illusionäre Transparenz ein. Daher hat der methodologischen Kritik an der Uneindeutigkeit der "Messergebnisse" eine Kritik an deren Präsentation an die Seite zu treten: Es entsteht ein krasses Missverhältnis zwischen Vagheit, Offenheit und Entscheidungsträchtigkeit bei der Datengenerierung und Eindeutigkeit, Präzision und Regelhaftigkeit bei der Datenpräsentation.

Fazit: Das Verfahren, mit dem Sobota zu ihren Ergebnissen einer Hintanstellung von Argumentation gegenüber Rhetorik kommt, hat einige Ähnlichkeiten mit empirischer Forschung. In der Hauptsache ist es ein Verfestigen von Interpretationsmöglichkeiten im Sinne mitgebrachter Einstellungen und Ambitionen. Die Forderung nach intersubjektiver Nachvollziehbarkeit der Resultate wird an den Stellen, an denen die Identifikation relevanter Textvorkommnisse (Logos oder Ethos-Argumente und Figuren) stattfindet, nicht erfüllt. 


\section{Schluss: Der wahre Kern in Sobotas Ansatz}

Nach so vielen Seiten Kritik und Verwerfung möchte ich den Leser mit dem Geständnis überraschen, dass ich Sobotas Bemühungen nicht in Bausch und Bogen ablehne, sondern dass ich in ihnen ein völlig richtiges bedenkens- und beherzigenswertes Motiv am Werke sehe. Es ist das Anmahnen der Historizität des Rechts, anders gesagt, die Veränderlichkeit des juristisch als richtig und gültig Angesehenen. Die Lebenswirklichkeit ändert sich und mit ihr die theoretische Basis des Rechts. Konkret gesprochen bedeutet das, dass sich unter dem Verfolgen von Zwecken durch Mittel die technischen und sozialen Bedingungen des Handelns und auch die Akteure selbst, also ihre Bewertungen und Einsichten ändern. Derartige Änderungen vollbringen wir nicht als Handlungen, über sie verfügen wir nicht, sondern sie widerfahren uns. Diese Widerfahrnisse zeigen sich nun insbesondere darin, wie argumentiert wird, letztlich darin, welche Argumentationen als richtig und gültig eingesehen und welche als nicht gültig verworfen werden. Eine Änderbarkeit des aufgrund von Argumenten als gültig Einsehbaren kann es aber nur geben, wenn der Begriff der Gültigkeit von Argumenten für so etwas offen ist. Das bedeutet, es muss später etwas als gültig bzw. ungültig einsehbar sein, was früher noch als ungültig bzw. gültig eingesehen worden war; und zwar nicht, indem Fehler berichtigt werden (das gibt es natürlich auch, ist aber der uninteressantere Fall), sondern indem sich die theoretische Basis der Argumentations- und Einsichtskompetenz verschiebt. Für die Jurisprudenz bedeutet das, dass der Normenkodex sich wandelt, indem den Normen bzw. den Begriffen innerhalb der bei der Beurteilung irgendwelcher "neuer" Fälle eingesetzten Normen eine neue Bedeutung zuwächst. Schlichter gesagt: Urteile, die eine neue Interpretation einer Norm als gültig begründen, sind "kreativ".

Wie sollen wir so etwas theoretisch darstellen? Solange Argumentieren deduktiv verstanden wird, kann über Argumente nur erreicht werden, dass das schon Gewusste und als gültig Eingesehene, vielleicht klarer, reproduziert wird. Dies ist leicht gesagt, hat aber eine gravierende Konsequenz: Ein logizistisches Verständnis vom Argumentieren muss an der Lebenswirklichkeit mit ihren Veränderungen und Entwicklungen scheitern. Eine Rechtstheorie, die daran festhält, muss lebens- und praxisfremd werden. Daher ist es richtig und sogar unabdingbar, über das (so verstandene) Argumentieren hinauszugehen.

Dieser zentrale Gedanke als das Hauptmotiv in Sobotas Arbeiten ist, wie gesagt, vollkommen richtig. Man kann sich nur wünschen, dass er anerkannt und verbreitet werde. Doch im nächsten Schritt beginnt So- 
bota zu irren bzw. ist sie einfach nicht genug über die neuen Anläufe in der Argumentationstheorie ${ }^{13}$ informiert. Mit ihrem Argumentationsbegriff befindet sie sich auf dem Stand von Aristoteles und verbindet dessen Theorieanläufe mit anderen, nicht zur Sache gehörenden Motiven (gegen den kantianischen Universalismus und gegen Habermas zu reden, die Rhetorik als Bildungsveranstaltung zu sehen etc.). Daher votiert sie dafür, über die Logik hinaus auf traditionale Bindung und rhetorische Beeinflussung zu setzen. Es ist dies aber kein Weiterkommen über die Logik hinaus, sondern ein Zurückfallen hinter sie, ein Zurückgreifen auf Methoden der Konsensfindung, die sich nicht an die autonome Vernunft wenden, sondern die auf schlichtes Überreden und Vereinnahmen hinauslaufen. Wenn durch den Einsatz rhetorischer Figuren oder durch Bezugnahme auf faktische Traditionen irgendetwas gültig erscheint, was sonst nicht so erschienen wäre, so ist es nicht gültig und richtig, sondern es scheint nur gültig und richtig.

Ein adäquater Argumentationsbegriff muss die Dynamik der argumentativen Prozesse erfassen können. Er muss das Argumentieren als ein sprachliches Handeln modellieren, das in nicht-sprachliche Handlungen eingebettet ist und bei dem es darum geht, gerade für die Situationen und Probleme, die "neu“ sind, Orientierungen zu generieren. Dazu ist ein Begriff von argumentativer Gültigkeit nötig, der sich jenseits der Alternative von logischer Wahrheit und rhetorischer Wirksamkeit artikuliert. Argumentieren ist das Ausweisen bzw. Kritisieren des Geltungsanspruches, welcher mit einer Behauptung erhoben wird. Es besteht nicht nur aus Gründen und Begründungsversuchen, sondern auch aus Einwänden und Widerlegungsversuchen. Eine These ist argumentativ gültig, wenn zu ihr eine Begründung konstruiert worden ist, die gegenüber den aktuellen und bekannten Widerlegungsversuchen (und nicht etwa gegenüber allen logisch möglichen Widerlegungsversuchen) gehalten werden kann. Solche Haltbarkeit ist also gleichbedeutend damit, dass es keine offenen Einwände gegen die These oder gegen irgendwelche Teile der Begründung (mehr) gibt. Formelhaft gesagt: Argumentative Geltung ist Einwandfreiheit. ${ }^{14} \mathrm{Ob}$ eine in diesem Sinne gültige These von einem bestimmten Auditorium akzeptiert wird, das ist eine Frage, die weitere deskriptive und normative Theorie über Strukturen in Orientierungsnetzen (Überzeugungssystemen, belief systems) und die Bedingungen ihrer Veränderung erfordert. (Dazu ist

${ }^{13}$ Vgl. Frans H. van Eemeren et Al. (Hrsg.), Fundamentals of Argumentation Theory. Mahwah (N. J.) 1996; STEPHEN TOULMIN, The Uses of Argument. Cambridge 1958; HARALD WOHLRAPP (Hrsg.), Wege der Argumentationsforschung. Stuttgart 1995.

14 HARALD WOHLRAPP, Argumentative Geltung, in: DERS. (Hrsg.), Wege der Argumentationsforschung. Stuttgart 1995, 280-305. 
bislang überhaupt nichts Sinnvolles zu lesen oder zu hören; und ob sich aus der traditionellen Rhetorik irgendetwas für diesen Zweck Brauchbares gewinnen lässt, das ist bis jetzt nicht abzusehen. Besonders optimistisch sollte man aber wohl nicht sein). Der so konzipierte Begriff von argumentativer Gültigkeit erlaubt es nun, und das ist der Gewinn gegenüber sowohl dem logizistischen als auch dem rhetorischen Ansatz, dass die über Argumentationen gewonnenen Einsichten sich entwickeln können. Wenn neue Sachverhalte neue Gründe oder neue Einwände gegenüber einer bislang als gültig bzw. ungültig eingesehenen These $\mathrm{zu}$ formulieren erlauben, dann kann die Integration derartiger neuer Argumente die Beurteilung der These ändern, ohne dass diese Beurteilung relativistisch wird. Mit einer solchen Konzeption lässt sich die Historizität des Rechts theoretisch abbilden. Die Rationalität des Rechts müsste nicht länger als ein wirklichkeitsfremder Mythos erscheinen. Vielleicht kann sich auch Sobota nach eingehender Prüfung und Reflexion davon überzeugen.

\section{Anhang}

Aus: BVerfGE 73, 339 (Solange II)

Beschluss des Zweiten Senats vom 22. Okt. 1986 - 2 BvR 197/83

B.II.1.a) Art. 24 Abs. 1 GG ermöglicht es, die Rechtsordnung der Bundesrepublik Deutschland derart zu öffnen, dass der ausschließliche Herrschaftsanspruch der Bundesrepublik Deutschland für ihren Hoheitsbereich zurückgenommen und der unmittelbaren Geltung und Anwendbarkeit eines Rechts aus anderer Quelle innerhalb dieses Hoheitsbereichs Raum gelassen wird (vgl. BVerfGE 37, 271 (280); 58, 1 (28); 59, 63 (90). Art. 24 Abs. 1 GG ordnet zwar nicht schon selbst die unmittelbare Geltung und Anwendbarkeit des von der zwischenstaatlichen Einrichtung gesetzten Rechts an, noch regelt er unmittelbar das Verhältnis zwischen diesem Recht und dem innerstaatlichen Recht, etwa die Frage des Anwendungsvorrangs. Innerstaatliche Geltung und Anwendbarkeit sowie ein möglicher innerstaatlicher Geltungs- oder Anwendungsvorrang völkerrechtlicher Verträge - auch der hier in Rede stehenden Art - folgen nicht schon aus dem allgemeinen Völkerrecht. Das gegenwärtige Völkerrecht enthält keine aus übereinstimmender Staatenpraxis und Rechtsüberzeugung entspringende allgemeine Regel dahin, dass Staaten verpflichtet wären, ihre Verträge in ihr innerstaatliches Recht zu inkorporieren und ihnen dort Geltungs- oder Anwendungsvorrang vor innerstaatlichem Recht beizumessen. Ein in- 
nerstaatlicher Geltungs- oder Anwendungsvorrang ergibt sich allein aus einem dahingehenden innerstaatlichen Rechtsanwendungsbefehl, und zwar auch bei Verträgen, die ihrem Inhalt zufolge die Parteien dazu verpflichten, den innerstaatlichen Geltungs- oder Anwendungsvorrang herbeizuführen. Art. 24 Abs. 1 GG ermöglicht es indessen von Verfassungs wegen, Verträgen, die Hoheitsrechte auf zwischenstaatliche Einrichtungen übertragen, und dem von solchen Einrichtungen gesetzten Recht Geltungs- und Anwendungsvorrang vor dem innerstaatlichen Recht der Bundesrepublik Deutschland durch einen entsprechenden innerstaatlichen Anwendungsbefehl beizulegen. Dies ist für die europäischen Gemeinschaftsverträge und das auf ihrer Grundlage von den Gemeinschaftsorganen gesetzte Recht durch die Zustimmungsgesetze zu den Verträgen gemäß Art. 24 Abs. 1, 59 Abs. 2 Satz 1 GG geschehen. Aus dem Rechtsanwendungsbefehl des Zustimmungsgesetzes zum EWG-Vertrag, der sich auf Art. 189 Abs. 2 EWGV erstreckt, ergibt sich die unmittelbare Geltung der Gemeinschaftsverordnungen für die Bundesrepublik Deutschland und ihr Anwendungsvorrang gegenüber innerstaatlichem Recht.

Aus: BVerfGE 65, 9 Zur Frage der ordnungsgemäßen Besetzung des Ersten Senats des BVerfG

Zwischenentscheidung des Ersten Senats vom 11. Okt. 1983 - 1 BvL $73 / 78$

Halbseite 155/2

a) Der Bundesrat hat e i n e $\mathbf{n}$ Bundesrichter in den Ersten Senat zu wählen.

Das in Art. 94 Abs. 1 Satz 2 GG, § 5 Abs. 1 des Gesetzes über das Bundesverfassungsgericht vom 12. März 1951 (BGBl. I S. 243) - im folgenden BVerfGG 1951 - statuierte Prinzip der paritätischen Berufung der Richter des Bundesverfassungsgerichts durch Bundestag und Bundesrat konnte ursprünglich sowohl für die Bundesrichter als auch für die anderen Mitglieder des Gerichts konsequent durchgeführt werden. Denn nach $\S 2$ Abs. 2, § 4 Abs. 1 BVerfGG 1951 waren in jeden Senat zwölf Richter, davon vier Bundesrichter, zu wählen. Durch das Gesetz zur Änderung des Gesetzes über das Bundesverfassungsgericht vom 21. Juli 1956 (BGBl. I S. 662) - im folgenden: Änderungsgesetz 1956 wurde die Zahl der Richter je Senat auf insgesamt acht, die Zahl der Bundesrichter auf insgesamt drei herabgesetzt (vgl. § 4 Abs. 1 Satz 1 BVerfGG in der Fassung des Art. 1 Nr. 3 des Änderungsgesetzes 1956). Das bisherige hälftige Verhältnis war daher nicht mehr durchführbar. 
Es wurde für die Bundesrichter durch das Verhältnis $2: 1$ ersetzt: $\S 5$ Abs. 1 Satz 2 BVerfGG in der Fassung

Halbseite 156/1

des Art. 1 Nr. 4 des Änderungsgesetzes 1956 bestimmte, dass ein Bundesrichter von dem einen, zwei von dem anderen Wahlorgan in die Senate gewählt werden. Das Gesetz hat jedoch nicht festgelegt, welche Körperschaft zwei und welche einen Bundesrichter zu berufen hat. Die Entscheidung darüber sollte vielmehr der tatsächlichen Entwicklung vorbehalten bleiben (vgl. Schriftlicher Bericht des BT-Rechtsausschusses über den Entwurf eines Gesetzes zur Änderung des Gesetzes über das Bundesverfassungsgericht, BTDrucks. II/2388, S. 2): Art. 3 Satz 1 des Änderungsgesetzes 1956 bestimmte, dass bis zur Verminderung der Zahl der einem Senat angehörenden Bundesrichter auf drei im Falle des Ausscheidens eines dieser Richter ein auf Zeit zu wählender Nachfolger berufen werden sollte, und zwar von demselben Bundesorgan, das den ausgeschiedenen Bundesrichter gewählt hatte. Dieser Fall trat beim Ersten Senat im Jahre 1958 ein, als der vom Bundesrat berufene Bundesrichter Wessel verstarb und von diesem Wahlorgan daher ein Richter auf Zeit als Nachfolger zu wählen war.

Halbseite 156/2

Das hatte nach $\S 5$ Abs. 1 Satz 2 BVerfGG in der Fassung des Art. 1 Nr. 4 des Änderungsgesetzes 1956 zur Folge, dass der Bundesrat künftig nur noch e in e $n$ Bundesrichter in den Ersten Senat wählen muss. An dieser Rechtslage hat sich durch $\S 5$ Abs. 1 Satz 2 BVerfGG in der Fassung des Art. 1 Nr. 4 des Vierten Gesetzes zur Änderung des Gesetzes über das Bundesverfassungsgericht vom 21. Dezember 1970 (BGBl. I S. 1765) - im folgenden: Änderungsgesetz 1970 - nichts geändert; der sachliche Inhalt der Vorschrift blieb unverändert. 